\title{
Allomaternal Nursing in Humans
}

\author{
by Barry S. Hewlett and Steve Winn
}

Few studies exist of allomaternal nursing in humans. It is relatively common among some cultures, such as the Aka and Efé hunter-gatherers of the Congo Basin, but it does not occur in other foragers such as the !Kung and Hadza of Southern and East Africa. This paper utilizes focal follow observations of Aka and Efé infants, interviews with Aka mothers, ethnographic reports from researchers working with hunter-gatherers, and a survey of the eHRAF cultures to try to answer the following questions: how often does allomaternal nursing occur, who provides it, and under what contexts does it take place? The study indicates that it occurs in many cultures $(93 \%$ of cultures with data) but that it is normative in relatively few cultures; biological kin, especially grandmothers, frequently provide allomaternal nursing and that infant age, mother's condition, and culture (e.g., cultural models about if and when women other than the mother can nurse an infant or colostrum taboos) impact the nature and frequency of allomaternal nursing. The empirical results of this exploratory study are discussed in the context of existing hypotheses used to explain allomaternal nursing.

Anthropologists have conducted several studies of breastfeeding from a variety of perspectives (Fouts, Hewlett, and Lamb 2012; Gottlieb 2004; Sellen 2007) and have occasionally described allomaternal nursing, that is, women other than mother nursing infants, but quantitative studies on the topic do not exist. Nutritional and health benefits of breast-feeding for mothers and infants are well documented (see American Academy of Pediatrics [2005] and Field [2005] for reviews), but what about infants who are breast-fed by women other than mother? Lactation is energetically costly, and allomaternal nursing may increase pathogen transmission between mothers and other infants. La Leche League, a global organization that promotes and assists women with breast-feeding, discourages both wet nursing (nursing another woman's infant, often for pay) and cross-nursing (the occasional nursing of another woman's infant while the mother continues to nurse her own child, often in a child-care situation), because the other women may transmit infectious diseases to the infant or cause the infant to be psychologically confused (Lawrence and Lawrence 2011; Minami 1995). Exceptions exist if the mother has health or other issues that lead to breast-feeding difficulties. ${ }^{1}$

Allomaternal nursing occurs in many cultures. Our review of cultures in the electronic Human Relations Area Files (eHRAF), a digital database of 258 cultures from around the world, found that it existed in 97 of 104 cultures with ethnographic data about

Barry S. Hewlett is a Professor in the Department of Anthropology of Washington State University, Vancouver (Vancouver, Washington 98686, U.S.A. [hewlett@vancouver.wsu.edu]). Steve Winn is Senior Vice President at the Behavioral Health Network (417 Liberty Street, Springfield, Massachusetts 01104, U.S.A.). This paper was submitted 8 VI 11, accepted 18 I 13, and electronically published 6 III 14. nonmaternal breast-feeding. It is somewhat expected in humans because, unlike the great apes, humans are cooperative breeders (Hill et al. 2011; Hrdy 1999; Ivey 2000; Kramer 2005; Turke 1988). Cross-species research indicates that allomaternal nursing is more likely to occur among cooperative breeders such as tufted capuchin monkeys (Cebus nigritus; Baldovino and Di Bitetti 2008) and sperm whales (Physeler macrocephalus; Gero et al. 2009). This paper focuses on allomaternal breast-feeding in hunter-gatherers because the authors know these groups best and have collected quantitative and qualitative breast-feeding data with foragers. This way of life also characterized over $90 \%$ of modern Homo sapiens history, and foragers lack the hierarchy and socioeconomic stratification common to most of the worlds' cultures today. We were also interested in understanding allomaternal nursing in cultures with diverse modes of production and social-political complexity and conducted the eHRAF survey mentioned above to see if forager patterns existed in other contexts.

The primary aim of our study is to understand the nature and contexts of allomaternal nursing in humans-how often

1. A popular form of allomaternal nursing in the United States is sharing expressed milk with adoptive mothers or mothers who have difficulties breast-feeding. Women use several cyber networks to obtain or donate frozen expressed milk with women who have low supply or are adoptive mothers. Also, milk exchange in the United States happens both informally and through established milk banks (with screening for transmittable viruses and such), and La Leche League promotes donations to milk banks. Milk donations used to be just for medical issues (i.e., donations to hospitals), but it appears that informal milk exchange is becoming more common. The milk banks can be problematic in Islamic countries with "milk kinship" beliefs because the milk donor is anonymous; the child could commit what would be perceived in the culture as incest if she unknowingly married somebody from the family of the woman who donated the breast milk. 
does it occur, who provides it, and under what contexts does it take place? We focus on empirical questions because few studies on human nonmaternal breast-feeding exist and we did not go to the field to test a specific hypothesis. Empirical patterns from our quantitative and qualitative hunter-gatherer research and eHRAF survey are described in the first half of the paper. The second half of the paper explores the applicability of existing hypotheses, mostly from the nonhuman animal literature, used to explain allomaternal nursing and considers alternative explanations for two unexpected results of the study-grandmothers were regular providers of allomaternal nursing, and nonmaternal breast-feeding disappeared in groups with normative allomaternal nursing by 12 months of age.

Various terms have been utilized to refer to nonmaternal breast-feeding. Wet nursing and cross-nursing are briefly described above. "Wet nursing" was the term most frequently utilized in our survey of the anthropological literature, but it was generally used to refer to any allomaternal nursing regardless of whether compensation was involved. Anthropologists also have used the term "cross-species nursing" to refer to situations where females nurse juveniles from another species (e.g., woman nurses a monkey), but it is also used to refer to nursing across different nonhuman species (e.g., a dog nurses a juvenile cat). Cross-nursing or co-feeding refers to mothers sharing breast-feeding or breast milk; a mother continues to breast feed her own infant and nurses another woman's infant a few times a day, often during exchanges of babysitting. "Allonursing" and "allosucking" are terms used by zoologists to refer to females that nurse or suckle offspring that are not their own. In this study we use the term "allomaternal nursing" to provide more precision to the term used in biology and to place it within the larger context of what is referred to as allomaternal care.

The paper is divided into three sections. First, we examine foragers and focus on our own quantitative data with Aka and Efé. Second, we turn to the eHRAF to identify broader patterns and evaluate whether forager patterns are consistent or inconsistent with cross-cultural descriptions. The eHRAF includes 258 cultures from all modes of production (e.g., foragers, pastoralists, farmers, market economies) and levels of socioeconomic stratification. Finally, we discuss the results in the context of existing hypotheses used to explain allomaternal nursing.

\section{Allomaternal Nursing among the Aka, Efé, and Other Hunter-Gatherers}

Three sources of data are utilized to answer the questions listed above: behavioral observations of Aka and Efé, interview data with Aka mothers, and a brief survey of ethnographers currently working with foragers. Methods for each data source are presented before results are described.

The Efé and Aka are both hunter-gatherers, often referred to as "Pygmies," in the Congo Basin. Efé are located in NE Democratic Republic of the Congo, speak a Sudanic language, number about 6,000, have social-economic relations with three farming groups, subsist primarily by bow and arrow hunting and providing labor to neighboring farmers, and have relatively low fertility (2.6 total fertility) and infant mortality (12.0\%). The Aka are located about 500 miles west of the Efé in the southern forest regions of the Central African Republic and northern Republic of Congo. They number about 35,000, have social-economic relations with at least 19 different farming groups, subsist by net hunting and trading with farmers, and have higher fertility (6.2 total fertility) and infant mortality $(20.0 \%)$ than the Efé. The two groups are similar in that age and gender egalitarianism and extensive sharing of food and child care (e.g., allomaternal care is extensive in both groups) are core values (see Hewlett 1996 for comparisons).

\section{Method}

Aka and Efé behavioral observations. As we will see in the eHRAF survey below, several ethnographers have noted the presence/absence and conditions under which allomaternal nursing may occur in a culture, but none of the eHRAF ethnographers provide quantitative data on its frequency and context. As far as we know, the Aka and Efé are the only cultures with quantitative data on allomaternal nursing. The data were collected as part of studies on infant development, and we were unaware of the frequency or nature of nonmaternal breast-feeding when we started the research.

Hewlett et al. (1998) conducted focal follow behavioral observations of 20 3-4-month-old and 20 9-10-month-old Aka foragers. Behaviors were coded every 30 seconds (20 seconds observe, 10 seconds record), and each infant was observed for about 9 daylight hours in both forest and village contexts. Winn conducted behavioral observations of 10 Efé infants at 3 weeks, 7 weeks, and 4 months of age. Behaviors were coded every minute, and each infant was observed for 2 hours at 3 and 7 weeks, and 4 hours at 4 months in the camp setting. Frequencies of allomaternal nursing are based upon the percentage or proportion of the total number of observation intervals allomaternal nursing occurred (e.g., if an infant spent .25 amount of time allomaternal nursing, it means that she was observed nursing in $25 \%$ of all 30 -second [Aka data] or 1-minute [Efé data] intervals). See Hewlett et al. (1998, 2000) and Tronick, Morelli, and Winn (1987) for more details on the codes and observational methods.

It is important to note that any time an infant was on the nipple of an adult female (not instances of a father or young teenage girl putting the infant to his/her nipple for a few seconds), including grandmothers, it was coded as "nursing" regardless of how much milk or fluid the infant actually received or whether or not they received anything at all. This is an important issue when considering the costs and benefits of nonmaternal nursing, and it is discussed in the second half 
of the paper. We have good reason to believe that infants usually receive something (fluid or milk) even if the woman is a grandmother, but we are not certain, so grandmother nursing might better be considered "probable" nursing.

Aka semistructured interviews. To determine whether the patterns that emerged from the behavioral observations were consistent or inconsistent with Aka views and feelings about allomaternal nursing, 10 mothers with infants less than 10 months old were interviewed. Mothers were asked whether any other female breast-fed their infants, contexts under which they think allomaternal breast-feeding occurs, whether they gave their colostrum to their infant, and ideas about who was an appropriate or inappropriate woman to breast-feed their infant.

Survey of ethnographers working with hunter-gatherers. We wanted to understand allomaternal nursing in forager groups other than Aka and Efé, so we contacted ethnographers and asked them about the existence and contexts of allomaternal nursing in the ethnic groups with which they were most familiar. The following ethnographers generously provided unpublished data: Tom Headland and Bion Griffin (Agta), Magdelena Hurtado (Aché), Vishvajit Pandya (Ongée), Nurit Bird-David (Nayaka), Paula Ivey Henry (Efé), Hillary Fouts (Bofi), Melvin Konner and Patricia Draper (!Kung), Russell Greaves (Pumé), Brooke Scelza (Martu Aborigines), and Peter Gardner (Paliyan). Bonnie Hewlett and Courtney Meehan also provided additional unpublished data on the Aka.

\section{Results}

Behavioral observations. Table 1 summarizes the frequency of nonmaternal breast-feeding among Aka and Efé. The Efé data at 3 and 7 months were combined due to the limited number of hours of observation per infant at each of these two age points ( 2 hours per age point) by comparison to 4 months ( 4 hours per infant).

How often does allomaternal nursing occur? The data indicate that allomaternal breast-feeding was common in early infancy among both the Efé and Aka foragers but declined substantially by late infancy among the Aka. The data indicate that when young infants receive allomaternal nursing, they spend, on average, $15 \%-25 \%$ of their total breast-feeding time with allomothers, and, in some cases, infants spend up to half or more of their nursing time with an allomother. While the amount of time allomothers breast-feed can be substantial, 7 of the 12 Aka infants who received nonmaternal breast-feeding spent less than $10 \%$ of their total breast-feeding time with allomothers.

The table also shows that the average number of Efé infants who received allomaternal nursing was similar for the two age points but that the average amount of time allonursing declined with infant's age. Allomaternal nursing rates among the Efé may be somewhat higher than among the Aka because the Efé camps have more adult women without infants who are available to allonurse. This is possibly due to lower total fertility rates among the Efé (2.6 live births for Efé women vs. 6.2 live births for Aka women); a high percentage of Efé women $(47 \%)$ have one child or less, primarily due to gonorrhea and other sexually transmitted infections (Hewlett 1996).

Aka allomaternal nursing declined substantially from early to late infancy. To determine what happens after late infancy we turned to other Aka and Efé field researchers who have conducted systematic observations with 1-3-year-olds. All researchers indicated that allomaternal breast-feeding was rare or disappeared by the time infants reached 12 months of age. Paula Ivey Henry (Efé), Courtney Meehan (Aka), and Hillary Fouts (Bofi) conducted observational studies with older infants and young children, and all reported that nonmaternal breast-feeding was rare or absent. Paula Ivey Henry did not observe one case of allomaternal nursing among Efé older infants and young children, Hillary Fouts observed one special case during her focal follows where the mother died, and Courtney Meehan identified only one case from her observational study - an Aka adolescent female offering her breast to a 1-year-old. Ivey Henry's quantitative behavioral observations are particularly instructive, as allomaternal nursing was so common in Efé early infancy.

In what contexts does allomaternal breast-feeding occur? This section examines behavioral data that provide information about the context of Aka nonmaternal breast-feeding at 3-4 months of age. Comparable data on Efé were not available. First, we compared the following behavioral measures of the 12 Aka infants who received allomaternal nursing with the 8 Aka infants who did not: frequency infant fussed or cried during observation hours, amount of time mother worked,

Table 1. Frequency of allomaternal nursing among Aka and Efé foragers

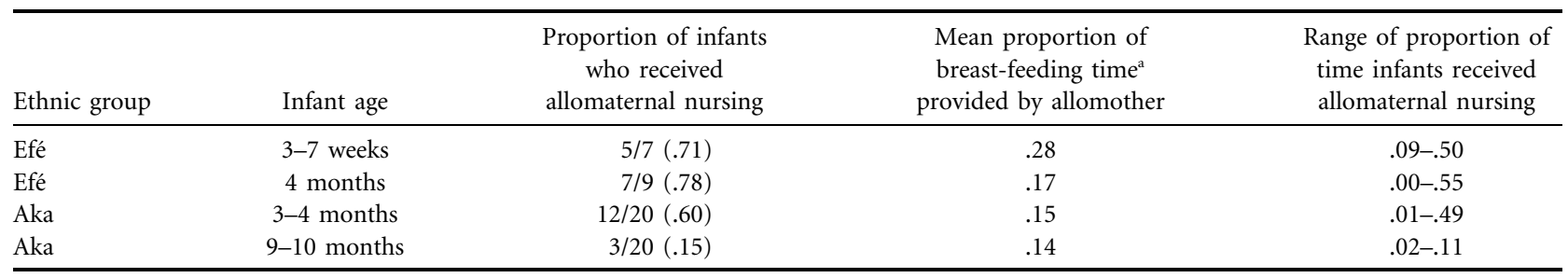

a Proportion of intervals behavior occurred during all observations. 
Table 2. Comparisons between 12 Aka 4-month-old infants who received allomaternal nursing and 8 4month-old infants who did not

\begin{tabular}{|c|c|c|c|c|c|}
\hline & $\begin{array}{l}\text { Infant received } \\
\text { allomaternal } \\
\text { breast-feeding } \\
\quad(n=12)\end{array}$ & $\begin{array}{l}\text { Infant did not receive } \\
\text { allomaternal } \\
\text { breast-feeding } \\
(n=8)\end{array}$ & $t$-statistic & $P$ (one-tailed) & df \\
\hline Proportion of time mother worked & .29 & .28 & .07 & .47 & 18 \\
\hline Proportion of time infant fussed or cried & .05 & .05 & -.01 & .50 & 14 \\
\hline Proportion of time mother held infant & .71 & .73 & -.02 & .41 & 17 \\
\hline Mean no. of minutes infant breast-fed ${ }^{a}$ & 81.6 & 84.0 & -.17 & .43 & 16 \\
\hline $\begin{array}{l}\text { Mean no. of minutes infant breast-fed } \\
\text { by mother }\end{array}$ & 70.5 & 84.0 & .96 & .35 & 18 \\
\hline
\end{tabular}

amount of time mother held infant, and minutes infant was breast-fed by mother. We thought infants who fussed or cried more, were held or breast-fed less frequently by mother, or had mothers who worked more would be allonursed more than other infants. Table 2 summarizes comparisons between Aka infants who received allomaternal nursing with those who did not. No significant differences existed between the two groups. Mothers who had nursing assistance from allomothers breast-fed their infants about 16\% less frequently, but the differences were not statistically significant.

Figure 1 examines the relationship between the amounts of time an Aka 3-4-month-old infant was held by an allomother and the amount of time the same infant received allomaternal nursing. The amount of time is the proportion of 30-second intervals in which the behavior (e.g., holding or allomaternal nursing) occurred. The relationship is highly significant $\left(n=12, r^{2}=.65, p=.001\right)$. Aka breast-feeding at 3-4 months is infant-initiated (i.e., as they sit on mother's lap or on her side, they take the breast on their own rather than mother or others deciding when to breast-feed) about $70 \%$ of the time (Hewlett et al. 2011). Among the Aka, allomaternal nursing is normative, giving/sharing is extensive, and infants are indulged (i.e., immediate response to fuss/cry and frequent breast-feeding), so it was not surprising that the longer a nonmaternal female held a young Aka infant the more likely she was to breast-feed the infant.

Figure 2 illustrates mother's activity while allomaternal nursing of her infant took place. Allomaternal nursing often occurred when mother was absent working, collecting firewood or water, but it also occurred about $40 \%$ of time when the mother was nearby (i.e., within a meter or two of the infant). When mothers were accessible to their infants, they were working (e.g., preparing a meal) about $50 \%$ of the time; the other $50 \%$ of the time an allomother nursed the infant while the mother was relaxing. Data also indicated that $51 \%$ of Aka allomaternal nursing bouts started without the infant fussing or crying. The second author did not code the same infant behaviors among the Efé, but his informal observations indicate similar patterns-allomaternal nursing took place when mother was working or relaxing, and it often took place without the infant fussing or crying.
Who provides the allomaternal nursing? Figure 3 examines the biological relationship between the infant and the allomothers who nursed the infants. The allomother was genetically related to the infant about $90 \%$ of the time, and no differences existed in the number of matrilineal or patrilineal female relatives who provided nursing $\left(\chi^{2}=.14,1 \mathrm{df}, p=\right.$ $.71)$ nor in the amount of time matrilineal or patrilineal relatives provided nursing ( $t=1.19,8 \mathrm{df}, p=.27)$. Age of Aka allomothers was also estimated, and $62 \%$ of them were over 45 , postmenopausal, and usually the grandmother of the infant.

Aka semistructured interviews. The Aka call breast milk "water of the breast" (mai na dibele). Nine of the 10 mothers reported that other women breast-fed their infants, and eight of the nine women who received allocare identified two or more other women who breast-fed their infants. Only one woman with a newborn did not report nonmaternal breastfeeding, but she said her baby would likely be nursed by another woman in the future. Most allomothers mentioned were biological kin of the infant- $44 \%$ were grandmothers (57\% paternal, $43 \%$ maternal) and $44 \%$ were aunts $(57 \%$ paternal, $43 \%$ maternal). Infants were reportedly breast-fed by nongenetic kin $12 \%$ of the time. While the women who provided allomaternal nursing were usually related to the infant, they were not biologically related to the mother $63 \%$ of the time.

Aka mothers generally said it was important to intimately know the women who breast-fed their infants. It was important for mothers to know the foods that an allomother was eating because if the woman ate a taboo (ekila) food, it could cause her infant to get sick and potentially die. A female graduate student and mother of two conducted the interviews with the first author, and the Aka mothers were asked if they would allow the graduate student to breast-feed their infants, and 9 of 10 mothers said they would not do it because they did not know her and were concerned about ekila.

Seven of the 10 women said they did not give their infants their colostrum right after childbirth because they felt the yellow color might have ekila. They expressed it into the fire (i.e., put their breast near coals from a fire so the heat would 


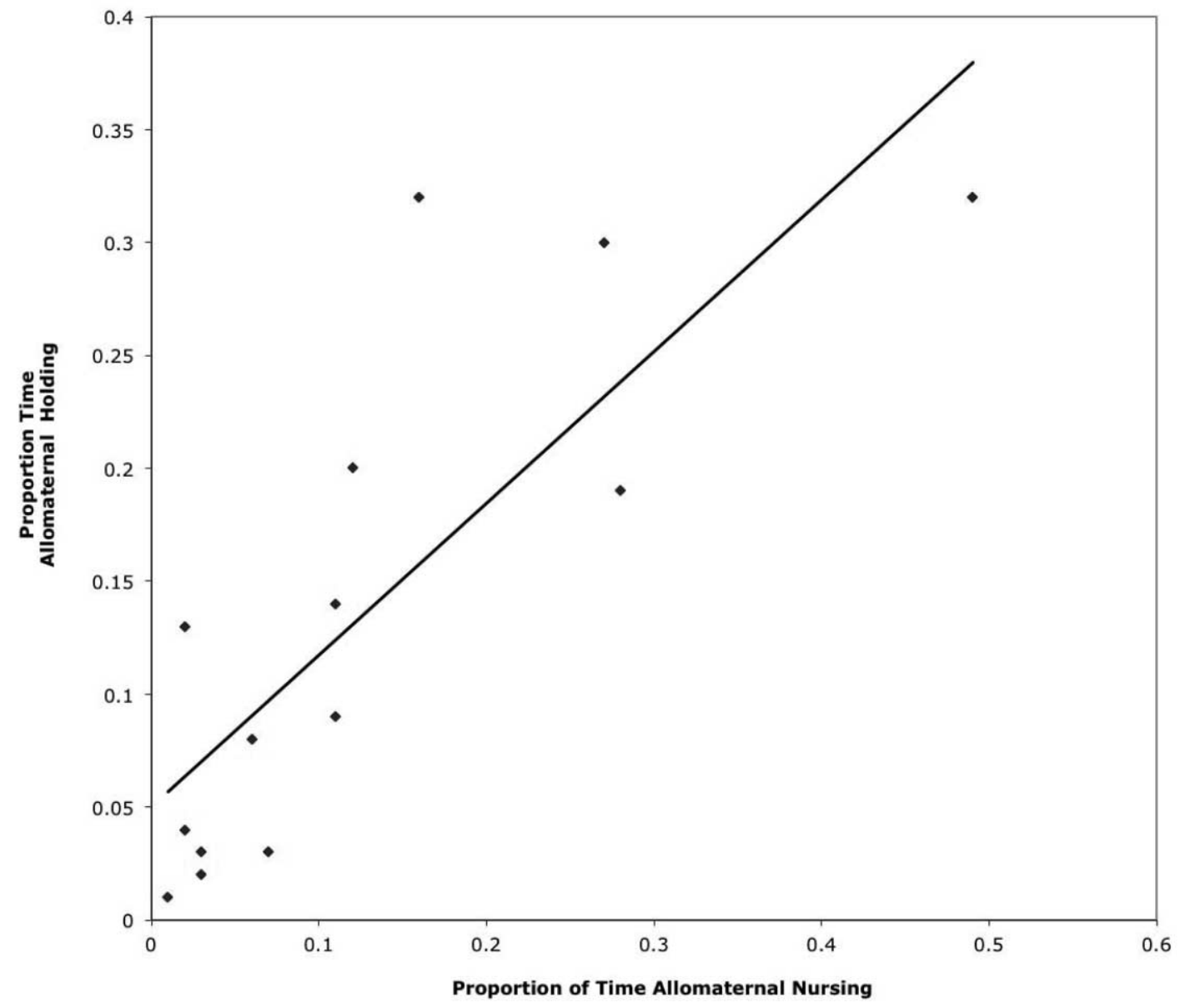

Figure 1. Relationship between frequency of allomaternal holding and frequency of allomaternal nursing among the Aka. Frequency is the proportion of 30 -second intervals in which the behavior occurred.

draw out ekila), fed their infant water, or another woman nursed their newborn until their milk arrived.

Aka infants have been observed attempting to nurse with their fathers, and fathers have been observed offering their nipple to fussy infants. We asked mothers about fathers trying to nurse and they said fathers may put the infant to their breast to try and soothe a fussy infant but they added that fathers are more likely to sing or dance with the infant or give her water before offering his breast. Mothers said fathers' nipples were too small and that the infants preferred the larger nipples of their grandmothers.

The interview data were inconsistent with the observational data in that mothers reported allomaternal nursing occurred much more frequently than we picked up in focal follows. This makes sense because each infant was observed for only 9 hours over a 3-day period. The interview and observational data were consistent in that allomaternal nursing was common; usually provided by close kin; and that grandmothers, both paternal and maternal, were particularly important contributors.
Allomaternal nursing among other hunter-gatherers. Table 3 summarizes the reports from forager ethnographers. Allomaternal nursing in infancy was normative, that is, not unusual, regular, or frequently observed among the Aka, Agta, Bofi, Efé, Chabu, and Ongée. Pandya says the Ongée word for woman is "milky breast"; if a woman leaves camp and another woman breast-feeds her infant, the mother says, "You have been a good milky breast." Not sharing a child is thought to be a bad quality in a woman. If a woman does not share her infant, others say, "Where does she think the child came from? It is for all of us." Griffin indicated that "of course" it is practiced among the Agta and that female hunters leave their babies in camp to be nursed by others. Headland, who worked with a different Agta group, says it is more likely to occur in emergency situations.

The frequency of allomaternal nursing varies in other forager groups. Among the Aché and Pumé, it reportedly occurs but not to the normative levels found among the Aka or Efé. Greaves reported several observations and contexts among the Pumé and among the Aché; Hurtado reported that "we 


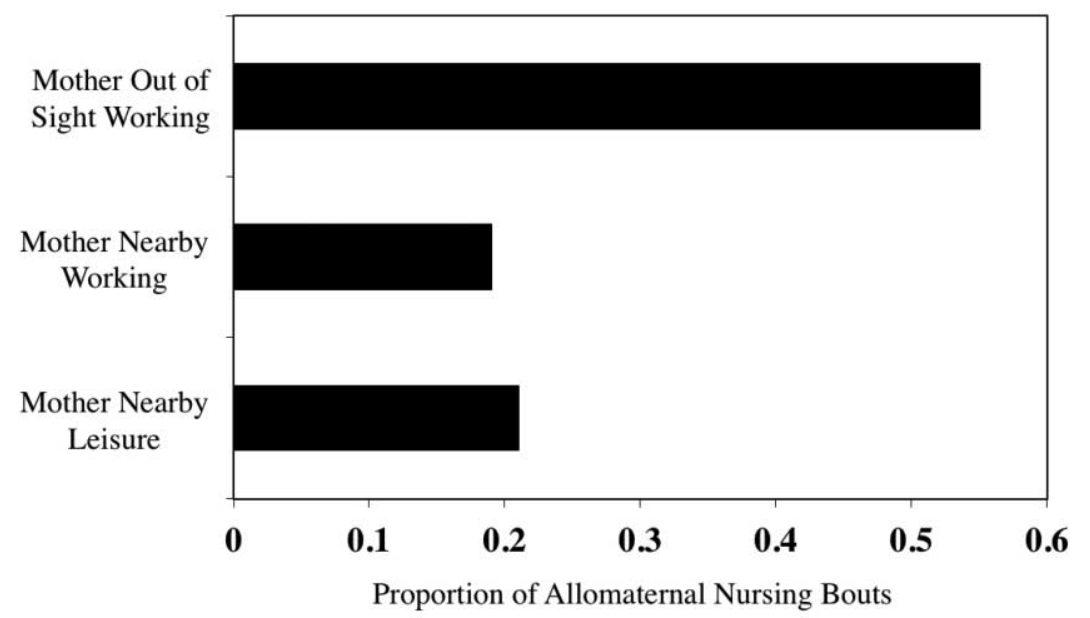

Figure 2. Aka mother's activity during allomaternal nursing.

know that it happens frequently enough that we observed several instances in the field." Allomaternal nursing was reported to be absent among the !Kung, Nayaka, Paliyan, Hadza, and rare among the Martu Aborigines. Konner stated "I never saw non-maternal breast feeding except once or twice as a sort of very brief joke." Bird-David never observed it among Nayaka, and Scelza described only one case among the Martu where a grandmother nursed her daughter's infant because the mother had a breast infection. Agta, Aché, and Nayaka ethnographers also reported that mothers breast-fed pets, such as monkeys. The data are too few to draw conclusions, but six of the seven forager cultures with normative allomaternal breast-feeding live in tropical forest environments, while it is absent or rare in the five cultures that occupy more arid environments.

\section{Cross-Cultural Patterns of Allomaternal Nursing}

Our quantitative data focused on hunter-gatherers, but we also wanted to explore nonmaternal nursing more broadly to try and determine whether the forager patterns were similar or different from a diverse range of cultures from around the world.

\section{Method}

Behavioral observations of Ngandu farmers. The Ngandu are the Bantu-speaking farming neighbors of the Aka. The Ngandu cultivate manioc, corn, plantains, and peanuts, and exchange some of their crops for meat and other forest prod-

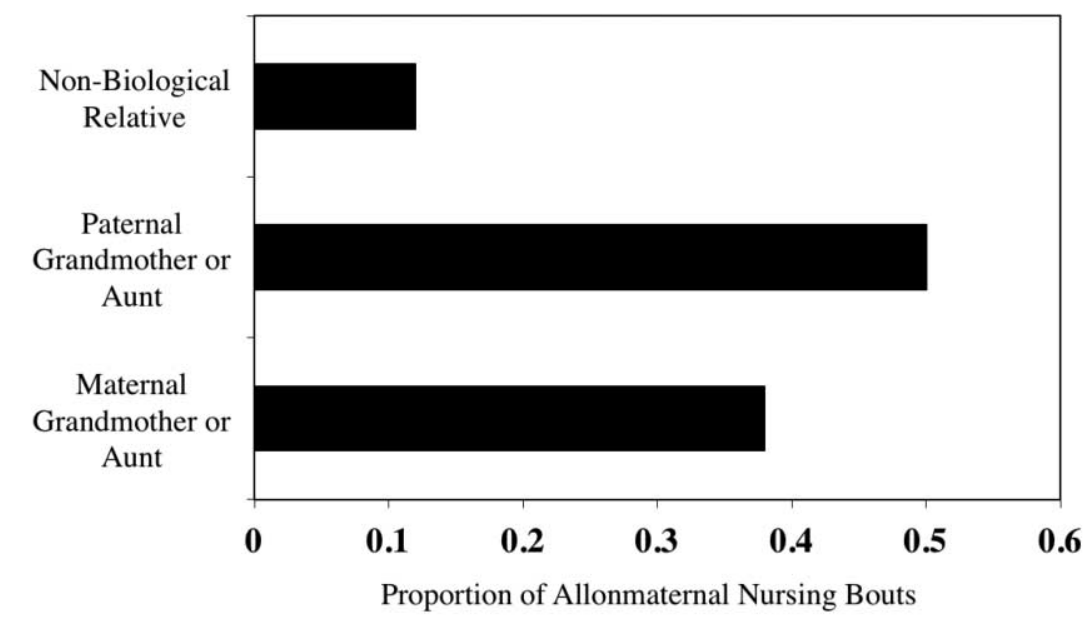

Figure 3. Genetic relationship between Aka infant and woman providing allomaternal nursing. 
Table 3. Infant allomaternal nursing in foragers

\begin{tabular}{llll}
\hline Forager ethnic group & \multicolumn{1}{c}{$\begin{array}{c}\text { Frequency of } \\
\text { allomaternal nursing }\end{array}$} & \multicolumn{1}{c}{ Ecology } \\
\hline Aka & Normative & Tropical forest & Reference \\
Efé & Normative & Tropical forest & Winn, this article \\
Bofi & Normative & Tropical forest & Hillary Fouts \\
Agta & Normative & Tropical forest & Bion Griffin and Thomas Headland \\
Ongée & Normative & Tropical forest & Vishvajit Pandya \\
Chabu & Normative & Tropical Forest & Barry Hewlett \\
Aché & Occurs & Tropical forest & Magdelena Hurtado \\
Pumé & Occurs & Wet savanna & Russell Greaves \\
Martu & Absent & Desert & Brooke Scelza \\
Nyaka & Absent & Dry savanna & Nurit Bird-David \\
Paliyan & Absent & Dry forest & Peter Gardner \\
$!$ Kung & Absent & Dry savanna/desert & Melvin Konner and Patricia Draper \\
Hadza & Absent & Savanna-woodland & Frank Marlowe and Alyssa Crittenden \\
\hline
\end{tabular}

Note. With the exception of the first two groups, references are based on personal communication.

ucts with the Aka. Women plant, maintain, and harvest the fields and provide the majority of the dietary calories, while men fish, hunt, and trade. Ngandu are patrilineal and patrilocal, number about 20,000, and have fertility and mortality rates similar to the Aka. Respect and deference toward elders (parents, older siblings) and males are core values among the Ngandu. The same focal follow behavioral observation methods described above for the Aka were also utilized with 20 3-4-month-old and 20 9-10-month-old Ngandu infants.

eHRAF survey. We conducted a survey of the 258 cultures in the eHRAF. We searched for all references to infant feeding (code 853) and key words wet nurse, foster mother, and milk mother. We found 208 cultures with data on infant feeding and key words, and 104 cultures with some ethnographic description or comments about allomaternal nursing.

\section{Results}

Ngandu farmers. Allomaternal nursing was rare among the Ngandu farmers who share the same natural environment as the Aka. The Ngandu believe that the breast milk of a woman other than mother is poisonous (ekila, or taboo) to an infant and will make her very sick and possibly die. Only one of the 20 Ngandu 3-4-month-olds infants was breast-fed by another woman, and none of the 9-10-month-olds received allomaternal nursing. The one case involved a grandmother nursing an infant during $11 \%$ of the infant's nursing bouts because the mother was very ill for several days.

eHRAF. Data on infant feeding existed for 208 cultures, and half of those cultures had data on allomaternal nursing. Table 4 summarizes the data by region of the world. All regions had data on allomaternal nursing, Middle Eastern and Middle American ethnographers were more likely to report it, and North and South American ethnographers were somewhat less likely to mention it. The ethnographic descriptions were often limited to a few lines, and no ethnography had quantitative data.
Table 5 summarizes the contexts of allomaternal nursing in the 104 cultures. The data are limited but indicate that it occurs in most cultures (93.3\%) and that seldom is it banned in any situation (e.g., even if mother dies). While allomaternal nursing was frequently mentioned in the ethnographic literature, it was normative in relatively few cultures $(n=6)$.

Allomaternal nursing was most likely to occur in emergency situations - the mother died, did not produce any or enough milk, or was ill. For instance, among the East Toradja of Sulawesi, "if the mother dies in childbed, or if for one reason or another she is unable to feed the child, then people look in the village or in the nearby vicinity for a woman who has a baby, and she is asked to feed the child along with her own" (Adriani 1951:559). Emergency situations were mentioned in a majority of cultures with data and are not unusual occurrences in "traditional" settings where maternal mortality and morbidity rates are relatively high and bottle-feeding is not an option.

Other than emergencies, allomaternal nursing occurs in three contexts-women in lower status group are required to nurse infants of the elite group, usually for some sort of compensation (i.e., wet nursing); women other than mother nurse a newborn for a day or so because the culture believes the mother's first colostrum is dangerous; other women nurse an infant while a mother is away from the village to travel or work. Wet nursing was particularly common in Eurasian stratified cultures (e.g., Hunza, Korean, Pashtun, and Malay cultures) but also existed in African kingdoms such as the Ashanti and Buganda.

The belief that colostrum is dangerous for newborn health is found in several parts of the world (Lozoff 1983), but only 10 cultures mentioned this in the eHRAF survey. For instance, among the Ibo of Nigeria: "For the first day or two after birth the mother does not nurse the child. The women declare that the first milk is 'bitter' and quite unfit for the child. . . . Custom forbids the mother from suckling it at this early stage; 
Table 4. Geographic distribution of eHRAF data on allomaternal nursing

\begin{tabular}{|c|c|c|c|}
\hline Geographic Area & $\begin{array}{l}\text { No. of cultures with data } \\
\text { on allomaternal nursing }\end{array}$ & $\begin{array}{l}\text { No. of cultures with data } \\
\text { on infant feeding }\end{array}$ & $\begin{array}{l}\text { Proportion of cultures } \\
\text { with data on infant feed- } \\
\text { ing that also had data on } \\
\text { allomaternal nursing }\end{array}$ \\
\hline Africa & 22 & 42 & .52 \\
\hline Asia & 24 & 42 & .57 \\
\hline Europe & 4 & 9 & .44 \\
\hline Middle America and Caribbean & 7 & 10 & .70 \\
\hline Middle East & 5 & 7 & .71 \\
\hline North America & 16 & 49 & .33 \\
\hline Oceania & 15 & 21 & .71 \\
\hline South America & 11 & 28 & .39 \\
\hline Total & 104 & 208 & .50 \\
\hline
\end{tabular}

instead, one of the old grandmothers or the midwife acts as proxy" (Basden 1966:59).

Some ethnographies described more than one setting for allomaternal nursing, and this was particularly true with cultures that practice "milk kinship"-that is, a cultural belief that an infant breast-fed by another woman becomes part of the allomother's kin group (usually the father's family or "milk fatherhood"). In this case, as the child grows older, he or she uses the terms "mother," "father," "brother," "sister," and so on, to refer to members of the allomother's family. This also influences the child's marriage options upon reaching adulthood because he or she cannot marry kin from the milk family. Fifteen of the 19 milk kinship cultures indicated that the allomaternal nursing occurred due to either the mother's death, insufficient milk, or because she was required to nurse an infant from an elite status woman. The label "milk kinship" (Khatib-Chahidi 1992; Parkes 2004) gives the impression allomaternal nursing is common, but our survey suggests it takes place under particular conditions and that it is not normative. Eleven of the 19 milk cultures are stratified, but ethnographers in only 5 of the 19 milk kinship cultures mentioned that lower class/caste women nursed infants from higher-status families. Milk kinship appears to be somewhat more likely in stratified cultures, but this does not mean that allomaternal nursing is always imposed on lowerclass women in these cultures.

Some eHRAF patterns were consistent with the huntergatherer results - early infancy is a particularly important time for allomaternal nursing, and biological relatives, especially grandmothers, often provide nonmaternal nursing. Contexts $1,2,3$, and 6 in table 5 are consistent with the forager result that early infancy is a particularly important time for nonmaternal breast-feeding. Mother's death during childbirth, the colostrum taboos, and mothers not able to breast-feed or not having enough milk emphasize the importance of allomaternal nursing in early infancy.

Table 5. Contexts of allomaternal nursing from a survey of eHRAF cultures (104 cultures with comments about allomaternal nursing)

\begin{tabular}{|c|c|c|}
\hline Contexts of allomaternal nursing & $\begin{array}{l}\text { No. of cultures } \\
\text { that mention }\end{array}$ & $\begin{array}{l}\text { Percentage of cultures } \\
\text { with data that mention }\end{array}$ \\
\hline $\begin{array}{l}\text { 1. Mother is not able to nurse or does not have enough } \\
\text { milk }\end{array}$ & 35 & 33.7 \\
\hline 2. Death of mother (usually in childbirth) & 30 & 28.8 \\
\hline $\begin{array}{l}\text { 3. "Milk kinship"—allomother's family become part of } \\
\text { child's kin network and impact child's marriage } \\
\text { choices }\end{array}$ & 19 & 18.3 \\
\hline $\begin{array}{l}\text { 4. Lower class/caste/clan women nurse infants of women } \\
\text { in ruling class/caste/clan }\end{array}$ & 19 & 18.3 \\
\hline 5. Mother is ill & 10 & 9.6 \\
\hline $\begin{array}{l}\text { 6. Allomothers nurse because of the belief that the } \\
\text { mother's first colostrum is dangerous to the newborn }\end{array}$ & 10 & 9.6 \\
\hline $\begin{array}{l}\text { 7. Allomothers nurse because mothers are working } \\
\text { away from home }\end{array}$ & 8 & 7.7 \\
\hline 8. Allomaternal nursing not allowed & 7 & 6.7 \\
\hline $\begin{array}{l}\text { 9. Allomaternal nursing common/normative (most infants } \\
\text { receive it) }\end{array}$ & 6 & 5.8 \\
\hline $\begin{array}{l}\text { 10. Allomaternal nursing exists but very limited ethno- } \\
\text { graphic description }\end{array}$ & 9 & 8.7 \\
\hline
\end{tabular}


Table 6. Individuals identified as providing allomaternal nursing in eHRAF survey

\begin{tabular}{lc}
\hline $\begin{array}{l}\text { Individuals identified as providing } \\
\text { allomaternal breast-feeding }\end{array}$ & $\begin{array}{c}\text { Percentage of cultures } \\
\text { that identified this indi- } \\
\text { vidual (no. of cultures) }\end{array}$ \\
\hline $\begin{array}{l}\text { Mother's or Father's mother (4 MM, } \\
\text { 3 FM, 6 no data) }\end{array}$ & $12.5(13)$ \\
Mother's or father's sister & $9.6(10)$ \\
Relative, not specific & $6.7(7)$ \\
Female relatives of mother, not specific & $6.7(7)$ \\
Any nursing woman available in the & \\
$\quad$ village or household & $9.6(10)$ \\
\hline
\end{tabular}

The eHRAF ethnographies seldom identified the allomother who provided the nursing, but when they did she was usually a biological relative; grandmothers, both maternal and paternal, were particularly important contributors. Table 6 summarizes the individuals identified as providing the breastfeeding. Of the 30 cultures where mother death was the reason for allomaternal nursing, grandmothers in 10 of the cultures were identified as the ones providing nursing. Nonbiological kin nursing infants was most likely to occur in stratified cultures where lower-status women nursed infants from elite families.

The eHRAF data were too limited to determine whether hunter-gatherers were more or less likely to practice allomaternal nursing than other societies. Eleven of the $104 \mathrm{cul}-$ tures with data were hunter-gatherers, and all had some data on nonmaternal breast-feeding, but only one of the six cultures with normative breast-feeding was a forager group.

Several of the cultural contexts identified in the eHRAF study also occur among foragers-allomaternal nursing during emergencies and delayed breast-feeding at childbirth due to colostrum taboos. In terms of emergencies, Hillary Fouts described a situation where a Bofi mother was ill and eventually died. An elderly female in the camp started to nurse the woman's 1-year-old infant on regular basis, and the child lived. Among the Martu aborigines, Brooke Scelza described how a grandmother in her 30s with her own infant started to nurse her older daughter's infant when the daughter got a breast infection and had trouble breast-feeding. The grandmother supplemented her daughter's breast-feeding until she recovered.

In terms of colostrum taboos, the Aka have this belief, and it occurs frequently (see Aka mother interviews above), while the Efé do not have the specific belief but have something similar. Efé mothers may give first colostrum to their infants but believe it is bure, or useless, and usually ask another woman to nurse the infant during the first few days after birth.

\section{Discussion: Explanations for Allomaternal Nursing}

This section places the quantitative and qualitative empirical descriptions of allomaternal nursing into theoretical contexts. We did not go to the field to test allomaternal nursing hypotheses, so it is not possible to provide a systematic test to reject or confirm specific hypotheses. It is important, however, to identify hypotheses currently used to explain allomaternal nursing and discuss whether or not they help us to understand why the observed variability exists. First, we describe six hypotheses for allomaternal nursing primarily from the nonhuman animal literature and then evaluate their applicability to the empirical descriptions. Second, we discuss possible explanations for two unexpected empirical patterns that emerged from the study: the importance of grandmothers in allomaternal nursing and the disappearance of allomaternal breast-feeding by 12 months of age in cultures with normative allomaternal breast-feeding.

\section{Existing Hypotheses}

Hypotheses to explain intracultural or intercultural variability in human allomaternal nursing are rare because few cultural anthropologists have conducted research on this topic. By comparison, evolutionary biologists have been interested in the topic for many years because females have been observed nursing non-offspring in at least 68 different species (Packer, Lewis, and Pusey 1992). These researchers are interested in identifying ultimate explanations for behaviors; that is, how a behavior promotes the survival and reproductive fitness of the individual, and they often start their analysis by considering the reproductive costs and benefits of the behavior. Table 7 identifies some of the potential costs and benefits of human allomaternal nursing. Both mother and infant increase their risk of exposure to infectious diseases from the allomother but obtain additional valuable energy-supplementary milk by the infant and reduced costs of lactation by the mother. Costs and benefits for individuals involved in nonmaternal breast-feeding will vary substantially by context. Factors may include (1) quality of allomother care, whether others are around to check allomother's care, age of allomother (e.g., juvenile females may learn to nurse/mother, older females may not have as much milk), (2) genetic relatedness of allomother to infant (i.e., more likely to risk costs if genetically related), (3) length of time allomother provides breast milk (e.g., costs for a female who takes over nursing after mother dies are much greater than for a female who does it briefly while mother is working), and (4) cultural norms about breastfeeding (e.g., sanctions for nursing another women's infant may increase costs or cultural norms that value it may increase social benefits).

Evolutionary biologists have proposed and tested several hypotheses in a variety of species, but we will not review all of them because several do not pertain to humans. For instance, allomaternal nursing is common in species that live in dense groups, such as seals and bats, but this may be due, in part, to the lack of efficient kin recognition, rarely an issue in human groups. Allomaternal nursing is also common in 
Table 7. Costs and benefits of allomaternal nursing ${ }^{a}$

\begin{tabular}{|c|c|c|}
\hline & Costs & Benefits \\
\hline Mother & $\begin{array}{l}\text { Increased risk of mortal- } \\
\text { ity or morbidity of in- } \\
\text { fant due to allomater- } \\
\text { nal care (injury, } \\
\text { neglect, infectious dis- } \\
\text { ease), increased risk of } \\
\text { infection }\end{array}$ & $\begin{array}{l}\text { Reduced costs of lacta- } \\
\text { tion, quick return to } \\
\text { ovulation and closer } \\
\text { birth spacing, more } \\
\text { rest and opportunities } \\
\text { to invest in subsistence } \\
\text { activities, more effi- } \\
\text { cient work effort }\end{array}$ \\
\hline Infant & $\begin{array}{l}\text { Increased exposure to in- } \\
\text { fectious diseases (via } \\
\text { breast milk, skin-skin } \\
\text { contact, respiratory), } \\
\text { risk of abuse/neglect }\end{array}$ & $\begin{array}{l}\text { Gain energetically rich } \\
\text { milk, emotional sooth- } \\
\text { ing, enhance immune } \\
\text { or probiotic system, } \\
\text { care if mother dies }\end{array}$ \\
\hline Allomother & $\begin{array}{l}\text { Energetic investment to } \\
\text { lactate, contribution to } \\
\text { survival of individuals } \\
\text { with unrelated genes } \\
\text { (if not related), in- } \\
\text { creased exposure to } \\
\text { infectious diseases }\end{array}$ & $\begin{array}{l}\text { Social, political, eco- } \\
\text { nomic, emotional alli- } \\
\text { ances with other fe- } \\
\text { males, inclusive fitness } \\
\text { (if genetically related), } \\
\text { enhanced immune sys- } \\
\text { tem? }\end{array}$ \\
\hline
\end{tabular}

a See Packer, Lewis, and Pusey (1992) and Roulin (2002) for overviews on costs and benefits of allomaternal nursing.

species with large litter sizes, again, another situation that does not pertain to humans or other primates (Packer, Lewis, and Pusey 1992).

The first five hypotheses in table 8 come from nonhuman research and are potentially useful for explaining human allomaternal nursing. The kin selection hypothesis proposes that females genetically related to the infant are more likely than unrelated females to provide allomaternal nursing. Support for this hypothesis comes from dwarf mongooses (Helogale parvula; Creel et al. 1991), gray mouse lemurs (Microcebus murinus; Eberle and Kappeler 2006), captive galagos (Galago senegalensis braccatus; Kessler and Nash 2010), and lions (Panthera leo; Pusey and Packer 1994). The grandmother hypothesis in anthropology (Hawkes et al. 1998) is an extension of the kin selection hypothesis in that it predicts that maternal grandmothers and aunts should be more likely than paternal kin to provide allomaternal care because of paternity certainty issues - that is, a woman's son and daughter-in-law may have an infant that is not genetically related to her. Other evolutionary biologists have argued that maternal kin should be more likely than paternal kin to invest in infants because paternal confidence of genetic relatedness is lower than maternal confidence (Alexander 1974; Daly and Weghorst 1982).

The empirical descriptions tend to support the kin selection hypothesis-figure 3 for the Aka and table 6 from the eHRAF survey-indicate biological kin provide most of the allomaternal nursing. About $90 \%$ of Aka women who provided allomaternal breast-feeding were genetically related to the infant. This was also true of the emergency cases provided by huntergatherer ethnographers; their stories described how grandmothers, sisters, or aunts supplemented or took over breastfeeding when the mother was unable or had difficulty nursing.
One may think that most of the individuals in a forager camp are biologically related to the infant, so it is not surprising that kin provide more of the allonursing, but a recent quantitative study of Aka and other forager camps shows that most adult individuals are not genetically related (Hill et al. 2011). Among the Aka, in a camp with an average of 16.6 adults, women have on average 1.5 primary kin (parents, brothers, sisters), and men have on average 1.9 primary kin in the camp. This means a husband and wife would have a total of 3.4 primary kin and 13.2 others available to help them with their infant; half of these adults would be female ( 1.2 female kin and 6.6 female non-kin). Primary kin provide $90 \%$ of Aka allomaternal nursing even though many other non-kin females are available to help out. Kin selection does not hold for most of the stratified and some "milk kinship" cultures because women from the lower class/caste/clan nurse infants of women from the elite or other social-political-economic group (Khatib-Chahidi 1992; Parkes 2004).

Hunter-gatherer and cross-cultural data do not support a matri-kin bias in allomaternal care as predicted by Alexander (1974), Daly and Weghorst (1982), and Hawkes et al. (1998). Aka behavioral and interview data indicated equal numbers of female matrikin and patrikin provided allomaternal nursing, and the cross-cultural data in table 7 is limited but suggests the same. The relatively equal amount of care provided by the Aka is likely due to their multilocal residence pattern. When mothers live with their families, matrikin provide allomaternal nursing, but when they live with their husband's family, patrikin provide it. Among the Efé and Ongée, two other cultures with high rates of allomaternal nursing, matrikin also do not provide much allomaternal nursing because they are virilocal not multilocal like the Aka, which means allomothers are likely to be patrikin and not related to the mother. Ethnographers Pandya (Ongée) and Winn (Efé) indicate this is the case. The matrilineal part of the grandmother hypothesis is not supported by our data, but the significance of grandmothers in providing allomaternal nursing in many cultures supports the basic premise of the hypothesis-that is, that grandmothers stop having offspring themselves and live longer so they can care for their grandchildren.

The second "learning-to-mother" hypothesis proposes that inexperienced females may be interested in nursing another female's offspring to improve their own maternal skills. Breast-feeding does not come naturally to mammalian species, and practice by adolescent or inexperienced females may increase their reproductive success. In many primate species, mortality of firstborn offspring is often higher due to mother's limited opportunities to learn to mother (Hrdy 1999; Lancaster 1971). Empirical evidence for this hypothesis in nonhumans is limited (Roulin 2002), and the same is true for humans. Most Aka allomaternal providers were grandmothers or adult women, few Aka adolescent females put an infant to their breast, and none of the eHRAF descriptions mentioned this. However, breast-feeding by adolescents does occur in some cultures because the second author found that grand- 
Table 8. Allomaternal nursing hypotheses

\begin{tabular}{|c|c|c|}
\hline & Hypothesis & References \\
\hline 1. Kin selection & $\begin{array}{l}\text { Females genetically related to the infant are more } \\
\text { likely than unrelated females to provide allomater- } \\
\text { nal nursing }\end{array}$ & Packer, Lewis, and Pusey 1992 \\
\hline 2. Learning to parent & $\begin{array}{l}\text { Juvenile females with breast development offer their } \\
\text { breast to suckle (no milk necessary) an infant to } \\
\text { learn how to nurse }\end{array}$ & Roulin 2002 \\
\hline 3. Reciprocity & $\begin{array}{l}\text { Two females obtain greater fitness by cooperating than } \\
\text { by nursing alone. Can occur immediately by shar- } \\
\text { ing nursing or delayed and indirect by providing } \\
\text { social, emotional, political, economic alliances }\end{array}$ & $\begin{array}{l}\text { Mann and Smuts 1998; } \\
\text { Packer, Lewis, and Pusey } \\
1992\end{array}$ \\
\hline 4. Imposition & $\begin{array}{l}\text { Elite families require low-status allomothers to nurse } \\
\text { their infants }\end{array}$ & Hrdy 1999 \\
\hline 5. Immunological & $\begin{array}{l}\text { Infants obtain more diverse specific immune com- } \\
\text { pounds from allomothers }\end{array}$ & $\begin{array}{l}\text { Roulin 2003; Roulin and } \\
\text { Heeb } 1999\end{array}$ \\
\hline 6. Cultural models & $\begin{array}{l}\text { Socially transmitted ideas, practices, and norms about } \\
\text { infant breast-feeding (i.e., learned expectations } \\
\text { about whether allomaternal nursing is allowed or } \\
\text { expected, and, if it is allowed, under what contexts } \\
\text { is it expected to take place) pattern allomaternal } \\
\text { nursing }\end{array}$ & $\begin{array}{l}\text { Fouts, Hewlett, and Lamb } \\
\text { 2012; Wells } 2006\end{array}$ \\
\hline
\end{tabular}

mothers seldom provided Efé allomaternal nursing and that young girls with any degree of breast development put the infant to their breast.

It is also interesting to note that the first author and Hillary Fouts have observed more Aka and Bofi infants on the breasts of their fathers than on the breasts of adolescent females. Aka and Bofi fathers hold their infants much more than adolescent females or Efé fathers (Hewlett 1996), so it is not surprising that Aka or Bofi infants may try to initiate nursing with their fathers.

The third hypothesis proposes that females should achieve greater fitness when they reciprocate nursing than when they do it on their own. Nonhuman animal studies have focused on situations where two or more lactating females sharing nursing and empirical support for this relatively symmetrical situation is limited because one lactating female in the group often ends up nursing more than others (Roulin 2002). Another form of reciprocity proposed by Mann and Smuts (1998) based on their work with bottlenose dolphins (Tursiops truncates) indicates females may nurse others' offspring to develop future social alliances.

Cooperation and sharing are part of human nature, so we assume reciprocity plays a significant role in the maintenance of allomaternal nursing in humans. Unfortunately, we have few data to say very much. Aka women did not talk about reciprocal nursing relationships, be it with kin or non-kin, and allomothers said they provided the nursing without any expectation of return. Many of the Aka and Efé women who provided allomaternal nursing did not have nursing infants. While rare, evidence of symmetrical reciprocity exists in the ethnographic record, such as in Manus where Mead (1930: 323) reports: "If the mother is ill and cannot entirely nurse her baby for some time, then she is expected to return milk to these wet nurses' babies if she gets her health back." The ethnographic record provides many more examples of the type of reciprocity proposed by Mann and Smuts, that is, females develop reciprocal social-emotional alliances, and allomaternal nursing is just one component of that relationship. Ethnographers in most of the societies that practiced milk kinship or imposed allomaternal nursing emphasized how, in addition to the gifts, jobs, or payments they received, it contributed to the development of social-economic-emotional alliances between families.

The fourth hypothesis proposes that members of the dominant group coerce females in the subordinate group to nurse their infants (Roulin 2002). Enforced allomaternal nursing of females of different dominance statuses occurs in a wide range of animals, such as wild dogs (Lycaon pictus), dwarf mongooses (Helogale parvula), and capuchin monkeys (Cebus capucinus; O'Brien and Robinson 1991). In some of the bestknown nonhuman cases of coercion, an alpha female kills all but a subordinate's infants, or else the pregnant alpha female kills the entire litter of subordinates and usurps milk for her own infants (Hrdy 1999). In some human groups, lower class/ caste/clan women are coerced, often with some form of compensation, by members of the elite class/caste/clan to nurse their infants. This has been described in Western history (Fildes 1988; Hrdy 1999) and is frequently reported in stratified cultures in the cross-cultural record (table 5). It is rare or does not occur in hunter-gatherer groups, in part due to their egalitarianism and extensive sharing. Researchers with both humans and nonhumans indicate lower class/caste women receive social-economic-political benefits for providing allomaternal nursing to the elite females. Cultural anthropologists have described the importance of the social-political dimensions of allomaternal nursing in some of the stratified "milk 
kinship" cultures (Khatib-Chahidi 1992; Parkes 2004). Their studies indicate that the elite benefit by relieving the mother of the energetic demands of nursing and enable her to have more children, while it benefits the allomother and her family by developing political, social, emotional, or economic relationships with the dominant status group (see cost-benefits in table 7).

The fifth hypothesis proposes that allomaternal nursing provides immunological benefits (Roulin 2003; Roulin and Heeb 1999) to the infant and allomother. Maternal milk contains antibodies or immunoglobins. Nursing another female's infant increases the frequency of nipple stimulation of the allomother, which increases or maintains prolactin concentrations that in turn provides increased immune protection to the infant. Nipple stimulation enhances prolactin production, which influences immunocompetence and immunological quality and quantity of milk in addition to reducing a female's fertility (Roulin 2003). This hypothesis indicates benefits to both allomaternal caregiver (e.g., prolactin production, increased immunocompetence, and suppression of fertility) and infant (e.g., immunological benefits, more milk). The authors also predict that allomaternal nursing would be more likely in young mammals with high parasite loads (especially macroparasites) or that are infected with virulent pathogens and that mothers should prevent allomaternal nursing when the risk of transmitting microparasites (e.g., viruses, bacteria) is high. They argue that even for women in the same pathogenic environment, different allonursers can provide infants with a stronger immune response, in part by supplying a different antibody (called "paratopes") from the mother for a particular pathogen.

Breast milk is a significant factor in the development and structure of the neonatal gut microbiota, and another woman's breast milk may optimize the young infant's immune system, especially in environments with diverse strains of bacteria (Martin et al. 2005). The hypothesis that allomaternal nursing contributes to the bacterial colonization of the young infants' digestive system is also worth considering.

Immune and microbacterial systems are complex, and considerably more ethnographic, behavioral, endocrinological, bacterial, and immunological data are needed to test the hypothesis. The only tentative support of either hypothesis in this study is that allomaternal nursing is more common among foragers living in tropical forest rather than arid environments: tropical forests have a greater diversity of infectious and parasitic diseases than arid environments (Dunn 1968). Allomaternal nursing was common or regularly observed among the Aka, Agta, Bofi, Efé, Ongée, Chabu, and Aché forest groups while it was absent or rare among the !Kung, Hadza, Nayaka, Paliyan, and Martu living in arid desert or savanna environments. But ethnographic data also raise some questions: Why do Ngandu farmers, who live in the same tropical forest as do Aka foragers, strongly discourage allomaternal nursing? Are sedentary farmers with larger villages and higher population densities at risk of more viral, virulent, or other infectious diseases that are not found among the Aka and that can be transmitted by breast milk? Aka and Ngandu have regular social-economic-ritual interactions, and macroparasite data indicate few differences in pathogen loads between the two groups.

We note that both Aka and La Leche League women are concerned about illness transmission through breast-feeding. Our interviews suggest that hunter-gatherer women have accumulated knowledge about the potential risks of allomaternal nursing, while La Leche League women utilize biomedical knowledge, but in both cases it suggests awareness and concern of illnesses transmitted by allomaternal nursing. If the immunological hypothesis is useful, the benefits may be greater than the costs for Aka allomaternal nursing women living in an environment where infectious and parasitic diseases are the primary causes of death (Hewlett et al. 1986). In contrast, the costs may be greater than the benefits for Euro-American women because they are at low risk of infectious or parasitic disease infection, but their infant may risk infection of an infrequent but virulent virus from another woman (e.g., HIV).

The final hypothesis proposes that socially transmitted and learned beliefs and values (i.e., cultural models) influence the nature and frequency of allomaternal nursing. The first five hypotheses are ultimate or fitness-enhancing explanations for allomaternal nursing, while this one is proximate, that is, an immediate cause or mechanism of the behavior that may or may not complement the ultimate cause. Several studies of breast-feeding have pointed out the importance of cultural beliefs and practices for understanding intercultural and intracultural variability (Fouts, Hewlett, and Lamb 2012; Wells 2006), and we assume this hypothesis applies to breast-feeding by women other than mother. Some cultures encourage allomaternal nursing, while others strongly discourage it or have sanctions against it. As described above, Ongée foragers highly value allomaternal nursing, while among the !Kung teasing is used to discourage it, and like the La Leche League, seven cultures in the eHRAF have cultural beliefs and sanctions to dissuade females from doing it. Culture also appears to impact Aka and Ngandu differences in allomaternal nursing-they live next to each other and interact with each other on a daily basis in the same tropical forest environment, but the Aka are one of the few cultures in the world where allomaternal nursing is normative while among the Ngandu it is prohibited.

How can we explain the enormous within and between cultural diversity in allomaternal nursing? We believe that, like most any human behavior, it is a matter of interactions between biology (e.g., fitness maximizing) and culture (i.e., social learning). Sometimes culture enhances reproductive fitness, and at other times it does not, and at other times it is independent and takes a course of its own, likely because it is not linked to fitness (Durham 1992). Cultural models that enable women other than a mother to nurse a baby if the mother dies, is sick, or does not produce enough milk, are likely fitness enhancing. The alternative caregiver is usually a 
biological relative, often a grandmother. Allomothers enhance their inclusive fitness by making sure the infant survives, and grandmothers often step up to nurse because the reproductive costs of nursing outlined in table 7 can be lower for older women with near completed fertility. These cultural models enhance the fitness of mothers, the infants, and the allomothers. On the other hand, seven cultures in the eHRAF survey did not allow allomaternal nursing even during these emergency circumstances, and the infants likely died. Cultural models in these instances may lead to fitness-lowering behaviors.

Cultural models in 10 eHRAF societies that prohibit newborns from nursing from their mothers because of the perceived dangers of the mothers' first colostrum may also be an example of how culture can lead to fitness-lowering behaviors. As previously mentioned, research has demonstrated the enormous benefits of mother's "liquid gold" (referring to the yellowish color of mother's first colostrum). Colostrum is rich in protein, antibodies, and immune cells, and is recommended for newborns by pediatric authorities. It is possible that adaptive explanations for these cultural models exist (e.g., removing accumulated contaminants or toxins from breast milk), but they have yet to be investigated. It may also be that the colostrum taboos are "neutral" in that they do not increase or decrease fitness because the mothers do not nurse for only 1 or 2 days, and the newborns eventually obtain their mothers' colostrum.

Cultural models that promote lower-status women nursing babies of elite women clearly enhance the reproductive fitness of the wealthy families because ethnographers describe the very high total fertility rates of elite women. Whether these cultural models contribute to the survival and fitness of poor families is not clear. As mentioned above, some ethnographers argue that low-status families gain social-economic alliances, but whether the practice enhanced the survival and fitness of the women who provided the breast milk has not been quantitatively evaluated.

The fitness-lowering impact of culture can also be seen in the European history of wet nursing, paying a woman to nurse an infant. After wet nursing had been practiced in France and Italy for 100 years, middle- and lower-class families started to adopt the cultural models of wet nursing because it was viewed as a status symbol. These families could not afford reliable and healthy wet nurses, and many of their babies died (Hrdy 1999). The poor families used a particular type of social learning that is adaptive in many contexts-copy the successful-but in this case it decreased rather than increased their fitness (Richerson and Boyd 2004).

Cultural models are an important factor for understanding allomaternal nursing in humans, and they may enhance, oppose, or be neutral in relation to reproductive fitness considerations (see also Durham 1992). At this point in time we are not able to explain much of observed cultural diversity, such as why seven cultures and La Leche League do not allow allomaternal nursing or why it is normative in six other cul- tures. Culture likely plays a role, but how it interacts with the particular living conditions and natural environments of these cultures is not clear. We do not have one systematic study of human allomaternal nursing.

The hypotheses listed in table 8 can also be divided into two general groups - those that address questions regarding the evolutionary origin of allomaternal nursing in humans and those who help to explain the maintenance or absence of allomaternal nursing in contemporary cultures. Kin selection, learning to parent, reciprocity, and the immunological hypotheses provide alternative explanations as to why allomaternal nursing evolved in humans while imposition and cultural models may help to explain the intracultural and intercultural diversity in allomaternal nursing.

Finally, table 8 lists only six of the leading hypotheses for allomaternal nursing. Two other less prominent and cited hypotheses are worth briefly mentioning because they may be useful for understanding human intracultural variabilitythe stressed infant and mother's workload hypotheses. The stressed infant hypothesis proposes that allomaternal nursing exists to protect or to soothe stressed infants (predator attack, conspecific aggression) and not to transfer milk to them (Cameron 1998). The mother's workload hypothesis comes from studies of breast-feeding in small-scale cultures and hypothesizes that when mothers' workload is high they are more likely to receive allomaternal assistance and nursing (Kramer and Ellison 2010).

The mother's work hypotheses received mixed support from data presented in this paper, while the stressed infant hypothesis did not find much supportive evidence. The mother's work hypothesis was not supported by a direct testAka mothers with infants who were nursed by allomothers did not work any more than other women. But it was also true that when allomaternal nursing did occur, mothers were likely to be some distance from the camp collecting firewood or water, or were working (e.g., preparing a meal) nearby in the camp. A direct test also did not support the stressed infant hypothesis - infants who received allomaternal nursing did not fuss or cry any more frequently than other infants. Observational data also indicated that $50 \%$ of allomaternal nursing bouts started without the infant fussing or crying. Winn, Morelli, and Tronick (1987) also found that young infants that fussed and cried more were less likely to be breast-fed by others.

In summary, the data in this paper provide the most support for the kin selection and cultural models hypotheses and the least amount of support for the learning to parent hypothesis. Substantially more data are needed to evaluate all of the hypotheses.

\section{Emerging Issues}

This section of the discussion addresses two unexpected issues that emerged during the study. We were surprised to find that allomaternal nursing in the Aka and Efé disappeared by the 
time infants were 12 months old even though the Aka infants continued to breast-feed until age 3 and the Efé infants to age 2. It was also surprising to find that Aka grandmothers often put infants to their breast and how often grandmothers were mentioned in the eHRAF survey as important contributors to allomaternal nursing.

Why does allomaternal breast-feeding disappear by late infancy? Aka and Efé allomaternal breast-feeding is common in early infancy, but it is absent or greatly reduced by late infancy. The importance of early infancy allomaternal breast-feeding was also evident in the eHRAF study. Is this due to attachment to specific others (i.e., mother), less crying for food as the infant gets older (human crying peaks during early infancy), slowing of brain growth in late infancy (peak velocity in early infancy), more supplemental foods can be consumed as the infant gets older, or that immune supplements from allomothers are not needed?

We are unable to answer these questions with existing data, but we hypothesize that attachment has at least a partial role in the change. Hunter-gatherer breast-feeding is often infantinitiated (Hewlett et al. 2011), which means that the lack of allomaternal nursing in older infants is likely due to decisions of the infant rather than the allomothers deciding not to nurse. Older infants seem to prefer to spend more time with mother; among the Aka the proportion of time infants are held by mother in camp increases from $51 \%$ in early infancy to $77 \%$ in late infancy (Hewlett 1991a). The dramatic decline in hunter-gatherer allomaternal nursing by 12 months of age is at least in part due to infant preference to nurse from a specific caregiver, that is, mother. Of course if unusual circumstances arise, such as mother's illness or death, allomaternal nursing can occur at any age.

Do postmenopausal grandmothers nurse? Are they pacifiers? When we observed grandmothers putting infants to their breasts, we initially thought they were pacifiers, but ethnographic cases, such as the Bofi case mentioned above, suggest this is not true. Hunter-gatherer ethnographers provided several cases where postmenopausal grandmothers were able to produce milk. Tom Headland provided a story of an Agta mother with four children who died of cholera. Her youngest was 3 months old, and her husband's mother (infant's paternal grandmother), age 58, took charge of the infant and nursed her for 2 years. When the grandmother first started to breast-feed, Tom said to her, "I cannot believe you can produce enough milk." In response to Tom's comment, "she promptly stuck her breast out at me and squirted milk on my shirt." The second author had a similar story of an Efé grandmother expressing milk on him when he questioned her ability to produce milk. Russell Greaves also conveyed a story of a Pumé postmenopausal woman demonstrating to him and his female field assistant by expressing milk that it is not necessary to currently have a child to be able to nurse.

Hillary Fouts described another case where a Bofi forager mother did not want to nurse her 2-year-old son anymore because she was divorced, had a new boyfriend, and wanted to be with him. The mother's mother, who was clearly postmenopausal, thought it was too early to wean, so she started to breast-feed him all of the time until he was weaned.

Finally, as part of a study of Aka women's breast milk, Bonnie Hewlett asked seven postmenopausal women to try to hand-express breast fluid/milk and then tested the content with a field analyzer. One produced milk with high fat content, three produced clear fluid, and three were not able to produce fluid. The last three unable to produce fluid were elderly women over 70 years old; none of them provided allomaternal nursing during behavioral observations, and none of the Aka women listed an elderly woman as providing breast milk to her infant.

A woman's ability to lactate without becoming pregnant or being a postmenopausal grandmother is not new to medicine (Slone 1956). Several studies on "relactation" exist, and the World Health Organization (1998) advises considering it in several contexts (e.g., case management of sick infants, infants with feeding problems, when an infant's mother is severely ill or has HIV). No significant differences have been found in breast milk produced during relactation or induced lactation and that produced after birth (Kulski et al. 1981).

The eHRAF survey also produced several descriptions of grandmothers' and other women's abilities to start lactation several years after their last child. Three examples are provided below.

I know a case of a woman who had suckled seven children of her own, evenly spread at about three years apart, and who then began to nurse the baby of her eldest daughter, who had died in childbirth. (Bemba of Zimbabwe, in Richards 1939:67)

Very often the infant's maternal grandmother adopts it and suckles it, though she herself may not have borne a child for years. The Wabena declare that any woman who has once had a child and who is not past her menopause can "bring back her milk" by quite simple treatment. (Bena of Tanzania, in Culwick 1935:390)

It is a general Lepcha belief that any woman who has once borne a child can produce milk spontaneously when a baby sucks; and this seems to be borne out by the fact that on occasion if the mother dies the grandmother will suckle the grandchild. I myself saw Kurma's mother-in-law suckling her granddaughter, though it was over twenty years since she herself had borne her last child. (Lepcha of Bhutan/ India/Nepal, in Gorer 1938:239)

Data suggest that at least some postmenopausal grandmothers can produce milk or fluid when they nurse their grandchildren. They may be pacifiers at times, such as when Aka fathers try to offer their breasts to pacify infants. Benefits for infants and mothers can be greater than the costs, especially in emergency situations. Benefits for grandmothers could also be great if the infant survives (inclusive fitness) or 
nursing leads to her obtaining greater prolactin production and immunocompetence as suggested by the immune hypothesis.

\section{Summary and Conclusion}

Few data exist on allomaternal nursing in humans, and this is the first paper to present quantitative behavioral data and a cross-cultural survey on the topic. The data are limited but provide an entry point to try to understand nonmaternal breast-feeding in humans. We focused on hunter-gatherers because of our own field experiences and quantitative data on foragers and the thought that findings from foragers may provide insight into human nature or at least into allomaternal nursing in cultures with minimal political-economicsocial stratification and hierarchy. But we also wanted to identify patterns of nonmaternal breast-feeding in diverse, small-scale cultures and conduct a cross-cultural survey. We were interested in trying to answer three basic questions: how often does it occur, who provides it, and under what contexts does it take place?

Humans are cooperative breeders, and it was therefore not surprising to find that allomaternal nursing existed in over $90 \%$ of the cultures with data and that, in cultures where it was normative and quantitative data exists, young infants received more than $20 \%$ of their nursing bouts from allomothers. But the ethnographic record also indicated that normative allomaternal nursing was relatively rare, occurring in only $6 \%$ of the cultures with data, and that it was most likely to occur during emergency situations-a mother's death, illness, or inability to nurse a newborn infant. Normative allomaternal nursing may be more common among hunter-gatherers because $46 \%$ ( 6 of 13) of personal communications with forager ethnographers indicated that it was regular to extensive.

Women who provided allonursing in most cases were genetically related to the infant-generally the infant's grandmother. Evidence of women not genetically related to the infant providing breast milk occurred in contexts of wet nursing, that is, lower class/caste women were paid or received some compensation for nursing the infants of unrelated elitestatus women.

In cultures where allomaternal nursing was normative, such as the Aka, it was most likely to occur before 4 months of age and when an allomother held an infant for a long period of time. Contrary to ecological predictions, infants who fussed more did not receive more allomaternal nursing, and women who worked more did not receive more breast-feeding assistance. Several of the contexts for allomaternal nursing identified in the cross-cultural survey also indicated that nonmaternal breast-feeding was particularly important in early infancy (e.g., mother dies in childbirth, not able to nurse, and colostrum taboos).

Several theories to explain allomaternal nursing, primarily from nonhuman animal studies, were introduced and dis- cussed. It was not possible to systematically test the hypotheses, but at least some data existed to support kin selection, imposition, and cultural model hypotheses. Considerably more data and systematic studies are needed to evaluate the hypotheses.

Our study had several limitations. First, we did not evaluate the amount or whether the infants actually received milk from the allomother. Our own observations, those of other ethnographers, and studies of relactation suggest infants usually receive something, but the quantity and quality of the milk received is not known. This is particularly important for evaluating reproductive costs and benefits of allomaternal nursing. Second, it is difficult for a general ethnographer, such as those in the eHRAF cultures or forager ethnographers who did not conduct focal follows of infants, to informally observe allomaternal nursing because it often takes place within a relatively short age range (before 4 months), and episodes are often brief. Consequently, the ethnographic record probably underestimates the frequency and contexts of allomaternal nursing. Ethnographers note its occurrence in emergency situations because these circumstances are often public and are brought to the attention of the ethnographer. Finally, the behavioral data were collected for a variety of infant development studies, and we did not set out to test specific allomaternal nursing hypotheses. We did not realize allomaternal nursing was common when we started the study. Consequently, the study has the limitations described above, and we do not have the necessary data to adequately test existing hypotheses.

Allomaternal nursing is common cross-culturally, but it is poorly understood, and no systematic studies exist. Our study was exploratory and raised more questions than it answered. Considerably more research is needed to test the immunological and other hypotheses. The results could have both basic research (understanding human nature and cultural diversity) and applied (public health, such as healthy contexts for allomaternal nursing) implications.

\section{Acknowledgments}

We sincerely thank and appreciate the tolerance of Aka and Efé families for allowing us to observe their daily lives. We thank the Central African Republic and Democratic Republic of Congo (Zaire at the time of research) for their support and authorizations to conduct the research. We are especially grateful to ethnographers who shared unpublished data on allomaternal nursing: Tom Headland and Bion Griffin (Agta), Magdelena Hurtado (Aché), Vishvajit Pandya (Ongée), Nurit Bird-David (Nayaka), Peter Gardner (Paliyan), Paula Ivey Henry (Efé), Hillary Fouts (Bofi), Melvin Konner and Patricia Draper (!Kung), Russell Greaves (Pumé), Brooke Scelza (Martu Aborigines), Bonnie Hewlett (Aka) and Courtney Meehan (Aka). We also sincerely appreciate Ahren Geilenfeldt's assistance with the eHRAF survey. Sarah Hrdy, Hillary 
Fouts, Adam Boyette, and Bonnie Hewlett read early drafts of the paper and provided insightful and useful comments.

\section{Comments}

\section{Alyssa N. Crittenden}

Department of Anthropology, University of Nevada, Las Vegas, Nevada 89154, U.S.A. (alyssa.crittenden@unlv.edu). 27 VIII 13

Barry Hewlett and Steve Winn provide the first cross-cultural investigation of allomaternal nursing. They offer a detailed review of ethnographic data, both published and personal communication from other anthropologists, and introduce the first, and only, quantified data on rates of allomaternal nursing, collected by the authors among the Aka and Efé. They conclude that this behavior exists in over $90 \%$ of populations, yet only $6 \%$ of populations practice normative allomaternal nursing. Several hypotheses to explain allomaternal nursing are reviewed, yet due to limited data, none of these hypotheses were tested. Selected data offered limited support for the kin selection, imposition, and cultural model hypotheses. Their results, although adding insight into human behavior and providing a noteworthy platform for future research, must be interpreted with some degree of caution.

The authors are forthright in their approach to this topic and acknowledge that they did not begin their project with a specific hypothesis in mind-rather, they focus on empirical questions by extracting relevant data collected from focal follows on general infant behavior. Although the authors should be applauded for providing the first quantitative data on allomaternal nursing, it should be noted that the authors did not collect the data in a systematic way to capture all of the relevant stages of development during infancy, which might impact the representation of allomaternal nursing frequency. They report behavioral observations of 203 -4-month-old and 20 9-10-month-old Aka infants and 10 Efé infants at 3 weeks, 7 weeks, and 4 months of age. The selection criteria for these age intervals remains unclear; they appear to be based on neither relevant developmental stages nor the onset of lactation. Most conspicuously absent are any data on the immediate or early postpartum period, when women are most likely to turn to some type of supplementation (DaMota et al. 2012). In some cases it can take up to a week postpartum for a mother's milk to come in, replacing her colostrum. Given the high prevalence of colostrum taboos cross-culturally (Morse, Jehle, and Gamble 1990) and the fact that the first few days after birth are the critical window for the establishment of breast-feeding (Holmes 2013), it is possible that rates of allomaternal nursing might be higher during the early postpartum period.

Milk production of allomothers and milk intake by infants are two additional areas where further clarification is needed. Hewlett and Winn acknowledge that not all instances of in- fants at the breast are actual suckling, but the rates of pacification versus milk intake remain unclear. Milk intake varies between infants and is largely controlled by milk supply and demand (Hinde and Milligan 2011); the amount of milk produced by a mother or allomother is not likely to be representative of the amount of milk that an infant ingests, making estimates of energy consumption difficult to determine (Miller et al. 2013). Among human biologists studying lactation, behavioral observations of breast-feeding are the least desirable measurement of infant milk intake because they are notoriously unreliable (Scanlon et al. 2002). The principal methods of milk volume estimation, that is, weighing of mother and infant before and after feeding and studies using doubly labeled water (Miller et al. 2013), may introduce methodological limitations to anthropologists working among smallscale societies but must nevertheless be addressed. Milk intake was only given cursory treatment in the discussion of the potential costs and benefits of allomaternal nursing-a key element of their argument (see table 7). The authors repeatedly stress that lactation is energetically costly and that allomaternal nursing may be linked to pathogen transmission and/or immune function - both arguments thus rely on the ingestion of breast milk. It was impossible for the authors to address these issues during data collection, given that they were not intending to focus on allomaternal nursing when they went into the field, but it is necessary to keep these limitations in mind when interpreting the data.

One of the most intriguing points raised by the authors is in regard to the finding that normative allomaternal nursing is most common among tropical foragers. My own work with mothers and infants among the Hadza hunter-gatherers of Tanzania supports the authors' claim that foragers living in arid environments have little to no allomaternal nursing. Hadza women, although claiming in emergency situations that it might occur, have neither reported nor been observed engaging in allomaternal nursing. The authors go on to suggest that allomaternal nursing in tropical environments, with higher rates of infectious and parasitic diseases (Sattenspiel 2000), might bolster the immune systems of infants. Although no data on immune function of infants is available, the Hadza do have comparatively low rates of parasitic disease transmission (Work et al. 1973). By emphasizing the potential relationship between allomaternal breast-feeding and disease transmission/immune function, Hewlett and Winn have highlighted fascinating avenues for future research.

\section{Hillary N. Fouts}

Department of Child and Family Studies, University of Tennessee, Knoxville, Tennessee 37996, U.S.A. (hfouts@utk.edu). 2 IX 13

Hewlett and Winn's article provides the first quantitative study of allomaternal breast-feeding among humans. Furthermore, they have compiled a substantial amount of qual- 
itative data on allomaternal nursing from the eHRAF and from ethnographers working with hunter-gatherers. Their article makes a substantial contribution to the anthropological study of infant feeding. As Hewlett and Winn acknowledge, they did not collect data to specifically address questions about allomaternal nursing but instead conducted this exploratory study using existing data. Notwithstanding the important contributions that this study makes, the study also brings to light a tremendous gap in the anthropological study of infant feeding, making the case for a targeted biocultural study of allomaternal nursing among multiple cultural communities in multiple ecological contexts.

Hewlett and Winn's data from the Aka and Efé are based on focal infant follows and provide an infant's-eye-view of the process of allomaternal nursing. This approach is quite useful in providing data on the care of infants that is not biased toward the involvement of one particular caregiver (e.g., mothers), as they observed any caregiver who nursed the infants. They also acquired Aka mothers' perspectives of allomaternal nursing through interviews. In future studies, it would also be worthwhile to identify allomothers who typically provide allonursing and interview them to understand their motivations for nursing infants who are not their offspring. Hewlett and Winn describe two anecdotes from my research among the Bofi foragers-one in which a mother had died and her infant was nursed by an elderly female and one in which a grandmother nursed her 2-year-old grandson after his mother weaned him in order to live with her new boyfriend. In both cases, I spoke with the women providing the allomaternal nursing, and both women explained that they felt it was necessary to provide the nursing in order keep the children alive. The grandmother of the 2-year-old explained to me that if she had allowed her grandson to be weaned so early (i.e., around 2 years of age) he would have certainly died. She explained that he was far too young to be weaned, and weaning children of his age causes diarrhea. In both cases, the allonursing women spoke only of the children's welfare and did not mention any other factors, including possible reciprocity. This is consistent with Hewlett and Winn's conversations with women who provided allomaternal nursing. Among the Bofi foragers and possibly other cultures with both prolonged nursing and normative allomaternal nursing in early infancy, the practice of allomaternal nursing may be closely tied to the cultural model of breast-feeding as fundamental to the survival of infants and young children. Although these examples are extreme cases (i.e., nonnormative), understanding the motivations of women who engage in normative allomaternal nursing would be beneficial in understanding the interplay and trade-offs between cultural and biological factors that allomaternal nursing involves.

Hewlett and Winn address an interesting conundrum: why are tropical forest foragers more likely to practice allomaternal nursing than foragers in arid environments? The authors point out that high exposure to disease (typical in tropical forest environments) alone cannot explain the prevalence of allomaternal nursing among tropical forest foragers, because tropical forest farmers, who have similar exposure to disease, do not typically practice allomaternal nursing. Cultural-evolutionary scholars have recognized that different cultural groups do not always address adaptive problems to the same reoccurring conditions in the same ways and that there is striking cultural variation among groups living in similar environments (e.g., Boyd and Richerson 2005; Heinrich and Boyd 1998). Arguably, humans meet adaptive problems in culturally relevant ways. Ethnographic research among tropical forest foragers and farmers has shown that foragers and farmers in these contexts tend to have very distinct cultural practices and values (e.g., Bailey and DeVore 1989; Fouts, Hewlett, and Lamb 2012; Hewlett 1991b). Thus, perhaps allomaternal nursing is being used by the tropical forest foragers, rather than the tropical forest farmers, because it not only meets an adaptive need (i.e., providing immunological benefits to both infants and women who allonurse) but also promotes existing cultural values of the groups, such as the extensive sharing and cooperation among the Aka and the Efé.

In sum, Hewlett and Winn's article exemplifies what is gained when anthropologists transcend subdisciplinary boundaries and use integrated cultural and biological theories and methods. Hewlett and Winn provide an insightful discussion by examining data on allomaternal nursing through both biological and cultural lenses and exploring the interactive nature of biological and cultural processes. Their discussion emphasizes how allomaternal nursing, like breast-feeding more generally, is fundamentally a biocultural process-providing caloric and immunological benefits and risks to infants and allomothers, as well as being embedded in cultural contexts, expressed in ways that are culturally relevant and tied to cultural models.

\section{The Potential Wonders of Other's Milk}

\section{Katie Hinde}

Department of Human Evolutionary Biology, Harvard University, Peabody Museum, 11 Divinity Avenue, Cambridge, Massachusetts 02138, U.S.A. (katiehinde@gmail.com). 20 IX 13

Breast-feeding has long been of interest to anthropologists (Macadam and Dettwyler 1995; Maher 1992; Van Esterik 2002) spanning biological, biocultural, sociocultural, medical, and archaeological subdisciplines. More recently biological anthropologists have begun to systematically investigate mother's milk from an evolutionary perspective (Miller et al. 2013), exploring the sources of variation in milk synthesis and the consequences for infant development. Hewlett and Winn wonderfully complicate our knowledge of lactation with the previously underappreciated yet relatively widespread practice of allomaternal nursing. In cases of maternal death, 
nonmaternal sources of breast milk may ensure infant survival. However, many of their examples suggest that "other's milk" was provided on occasion rather than permanently replacing mother's milk. Allomaternal nursing may have importantly contributed to infant development and well-being, but little empirical data at present allow for such a conclusion. This preliminary discussion of allomaternal nursing should motivate further research that incorporates the physiological and social aspects of other's milk across cultures.

A number of physiological nuances of lactation biology are important to incorporate whenever considering the complex fluid that is milk, particularly in the context of allomother's milk. Hundreds of bioactive constituents are present in milk. These include fats, carbohydrates, proteins, minerals, vitamins, hormones, immunofactors, and water (Hinde and Milligan 2011), all of which vary among mothers and are therefore likely to vary among allomothers as well. As such, allomaternal milk in many cases may be a mismatch with mother's milk. For example, hormones in milk contribute to infant metabolism, neuroendocrinology, and behavior (Hinde 2013), and the effects of "mixed signals" from nonmaternal milk sources are entirely unknown. However, in some cases this mismatch may be beneficial to infants. Immunofactors in milk reflect pathogens encountered during the allomother's lifetime-not just immune challenges in the current environment shared by all community members. Notably, Hewlett and Winn report that a substantial proportion of allomaternal nursing is provided by the infant's paternal grandmother. This enables intergenerational transfer of immunological defenses against pathogens never encountered by the mother or maternal grandmother. Similarly, allomaternal nursing allows for the vertical transmission of beneficial commensal bacteria (Funkhouser and Bordenstein 2013) and oligosaccharides that feed that bacteria (Martin and Sela 2013).

The nutritional value of allomother's milk, however, remains uncertain. In a large study of induced lactation among adoptive mothers $(N=240)$, "nearly all" infants required supplemental formula to sustain growth (Auerbach and Avery 1981), indicating that induced lactation and relactation milk does not satisfy nutritional needs. Moreover, only $4 \%$ of these adoptive mothers reported lactational ammenorhea, suggesting that nipple stimulation is not sufficient to suppress cycling (see also Valeggia and Ellison 2009). In cases of grandmother relactation, gross milk composition (total fat, protein, and carbohydrates) is very much an open question. A single report by Gindler and colleagues (1985) shows that for a postmenopausal grandmother in Nigeria, milk protein concentration was $60 \%$ lower and fat concentration was $40 \%$ lower than typical breastmilk. However, allomaternal milk, even at lower nutritional value, can be an important "stopgap." Jonathon Wells has proposed that an infant adjusts metabolism in response to signals of the mother's embodied resources for lactation-such as fat stores and skeletal calcium (Wells 2007, 2011). Long internursing intervals likely influence metabolic processes. The infant's body, perceiving "food insecurity," may prioritize storage of abdominal fat over lean tissue or brain development with long-term consequences. Notably, Hewlett and Winn reveal that allomaternal nursing in hunter-gatherer cultures often occurs when the mother is working away from the infant. In this way allomaternal breast-feeding shortens internursing intervals and signals milk security. This leads to the prediction that allomother's milk improves metabolic development during early life, the effects of which may persist long after infancy.

Unfortunately, allomaternal nursing has not been a target of systematic research in any anthropological subfield, despite the numerous hypotheses in the literature. Given the dearth of information on this topic, we should be cautious about the incredibly seductive speculation of a cultural dichotomy between arid and forest ecologies. Hewlett and Winn highlight the Ngandu farmers as sharing a disease ecology with the Aka but not the practice of allomaternal nursing. Another counterpoint comes from interviews with Himba pastoralists in semi-arid Namibia. In that culture, some sisters and grandmothers will provide allomaternal nursing even though cow and goat milk are readily available (Scelza and Hinde, unpublished data). Further study of allomaternal nursing is necessary so that we can have a better understanding of the cultural, ecological, and situational contexts in which it occurs and the outcomes for mothers, infants, and allomothers. For better or worse, the greater availability of commercial infant formulas may have directly reduced the prevalence of allomaternal nursing throughout much of the world. Anthropological studies on this topic, as for so many other topics, should be pursued while living memory of traditional practices remains.

\section{Paula Ivey Henry, Gilda A. Morelli, and Edward \\ Z. Tronick}

Department of Social and Behavioral Sciences, Harvard School of Public Health, 677 Huntington Avenue, 6th floor, Boston, Massachusetts 02115, U.S.A. (piveyhen@hsph.harvard.edu)/Department of Counseling, Developmental, and Educational Psychology, Boston College, Chestnut Hill, Massachusetts 02467, U.S.A./Department of Psychology, University of Massachusetts, McCormick 271, 100 Morrissey Boulevard, Boston, Massachusetts 02125, U.S.A. 14 X 13

In 1993, a young Efé hunter-gatherer mother, Sikalu, died following the birth of her second child. She had first appeared in the Harvard Ituri Project census as an adolescent in a camp dense with kin, where she remained after marriage and the birth of her first child, Matu. As other Efé infants, Matu received intensive care from his mother, and many others. Years later, Sikalu gave birth again, and the newborn was passed and allonursed in the days postpartum. The mother never recovered from the complicated birth, and after she died, finding milk for the baby became difficult. The father promised to hunt and share food in 
exchange, but no woman was willing to nurse the failing newborn. The infant died, and in his grief, the father left. Matu remained in the attentive care of maternal kin. ${ }^{2}$

Contrasts in care illustrated by this case mirror challenges imbedded in Hewlett and Winn's review of human allonursing. Cooperative reproduction dominates the stage of the human evolutionary story, but ecological, biological, and cultural dimensions of shared investments remain unclear. How can we reconcile an Efé father's futile search for allonursing in a culture remarkable for the extent of its practice? Mother and child were surrounded with female kin, strongly predictive of allocare (Briga, Pen, and Wright 2012; Sear and Mace 2008), and the father offered the most valued resources, but neither "emergency" nor "normative" care was forthcoming. The contrast between Efé as an allonursing culture and the actual limits of its use exposes the significance of that threshold where human dependency can override the best social capital or most cooperative expectations of care. How far do humans push cooperation?

Hewlett and Winn open an entirely novel conversation about care and exchange that presses for research attention to measures of ecological constraint and to the social opportunities they provide (Hatchwell and Komdeur 2000). Although the paucity of quantitative inter- and intra-population data frustrates a human model of allonursing, it should encourage others to the freshly beaten path. Our comments explore analytical challenges allonursing poses and consider merging processes at play among Efé hunter-gatherers as suggestive of lines of inquiry in other populations.

The reproductive advantage of human life-history stems from a dramatic relaxation of primate quality-quantity tradeoffs, with robust benefits to parents and offspring wholly disproportionate to often nominal (and distributed) costs borne by providers (Ivey 2000; Lancaster and Kaplan 1992; Reiches et al. 2009). In fact, the social benefits of helping when most needed-especially by those most dependentcan more than offset the energetic and opportunity costs of aid (Kramer and Greaves 2011). Allonursing potentially changes those stakes, as milk is costly to produce and appears the perfect defendable resource (Dettwyler and Fishman 1992; Sellen 2007). Allonursing raises questions in addition to donor interests. Lactation is the most intimate maternal-offspring link postpartum, influencing a range of outcomes across the life span (Hinde and Milligan 2011; Lanigan and Singhal 2009), and attendant behaviors produce epigenetic and intergenerational modifications (Champagne 2011; Meaney and Szyf 2005.

The convergence of processes warrants clarification of hypotheses at proximate, adaptive, ontogenetic, and phylogenetic levels (Tinbergen 1963), and empirical delineation of

2. For project description, see Bailey and DeVore (1989). Names and identifying details have been changed. resources shared-milk, time, skill-in the context of other behaviors. Is allonursing an additive or replacement resource for maternal milk? Is it performed within the continuous flow of specialized handling that youngest infants require, or do mothers specifically pass their infants to allonurse? A prioritizing question is the extent to which lactating women allonurse. If grandmothers nurse children and grandchildren, inquiry can be directed to hunter-gatherer experience of overlapping generations; if not, to recurrent lactation; if not milk, to pacification success. Where data exist, analyses of maternal and infant condition, setting, demography, and relationship continuity (Fouts and Brookshire 2009; Morelli, Ivey Henry, and Foerster, forthcoming) and a directed energetic view (Meehan, Quinlan, and Malcom 2012) could help discriminate effects on mothers, infants, and others.

Missing cross-cultural and known intracultural allonursing variation requires cautious use of the term "normative." The authors make the emic case for expectations and prescriptions of allonursing among Aka, but the construct "normative" obscures rather than clarifies the phenomenon it seeks to describe. How much population or individual behavior warrants the label? Is the distribution of behavior or its cultural promotion to be explained? The heterogeneity that renders ecology and behavior so complex cannot be avoided (Evans et al. 2013; Sear and Gibson 2009), necessitating concepts that anticipate variation to match. Given the salient evidence of allonursing among African pygmies, we revisit Tronick, Morelli, and Winn's (1987; 1989) initial hypothesis of Efé allocare as a dynamic constellation of behaviors tailored to maternal and infant needs during a period of highest risk for both.

\section{Is Efé Allonursing a Maternal Strategy?}

Risk is an important driver of food sharing but is far less examined for care. Reproduction is not only costly but dangerous. Maternal mortality is high in traditional communities (Gurven, Kaplan, and Supa 2007; Hewlett 1991a; ZureickBrown et al. 2013), and body size, nutrient intake, and pathogen exposure are independent predictors highly relevant to the Efé (Dietz et al. 1989; Dormitzer, Ellison, and Bode 1989; McDermott, Steketee, and Wirima 1996; Zerfu and Ayele 2013). Efé allonursing follows the stress of pregnancy and childbirth when energetic balance is at its lowest (as during illness; Ellison 2001; Piperata 2008), and Efé women are responsible for the majority of calories (Bailey and Peacock 1988). Even limited assistance is likely to impact somatic repair and reserves. Efé mothers receive much more.

Adaptations during peak demands can be especially informative of local trade-offs (Jasienska 2009). In the absence of alternatives for milk or comfort, breasts do both, and bout frequency (maternal interruption) is highest in the first months postpartum. Consistent with Hewlett and Winn, longitudinal observations of Efé infants 4 months to 2 years old (from 18 bands over 20 months) found no allonursing later in infancy (Ivey 2000; Tronick, Morelli, and Ivey 1992). Lim- 
ited nonmaternal offers $(n=6)$ were associated with fussing and heavy maternal work (e.g., digging yams, chopping wood), and a teen attempting to nurse in play, which infants ignored or refused. While scant, they illustrate the use of another's breast as a means of both appeasing infant demands and extending mothers' work, tensions apparent in this review.

The interdependencies that favor Efé allocare may create reciprocal feedbacks sufficient to dampen maternal competition and foster allonursing. Intense perinatal support, at least for some Efé women, appears to extend through recovery and full resumption of work. However helpful, allonursing may not be long affordable. Nursing mothers have other demands: on average they support 2.5 weaned children in addition to their own infant $(n=144)$.

\section{Is Efé Allonursing a Developmental Strategy?}

Ontogenetic processes by which care is translated into phenotypes (Flinn et al. 2011; Lester et al. 2011) offer a bridge between cooperative views of reproduction to its reciprocal, cooperative development (Morelli, Ivey Henry, and Foerster, forthcoming). Hewlett and Winn's review should stimulate exploration of developmental experience and allonursing, including perinatal and postnatal metabolic and neurophysiological risks (Cowett and Farrag 2004; Dunsworth et al. 2012; Neubauer and Hublin 2012).

Efé allonursing is concentrated in the first 3 months of infancy, a period cross-culturally of peak crying, frequent feeding, continuous holding, and delicate care (Barr et al. 1991; Douglas and Hill 2011). Efé also experience unique constraints. Infant survivorship and development is strongly predicted by size (McCormick et al. 2006), and Efé have the smallest average birth weight recorded $(2.4 \mathrm{~kg}$, less than the fifth percentile). In spite of established risks, neonates show none of the expected decrements in neurobehavioral performance observed for small-for-gestational-age Western infants. In fact, they exhibit higher activity levels and lower state lability than expected for their size. Intriguingly, the Efé Ponderal Index at birth is greater than the ninetieth percentile (Tronick and Winn 1992), suggesting enhanced adaptation to postnatal demands (Kuzawa 1998).

Motor, social, and neurobehavioral variation emerges early in development in response to specific care behaviors (Morelli, Ivey Henry, and Forester, forthcoming; Super and Harkness 2008). To the extent Efé are exposed to allonursing, developmental patterns should reflect variation identified in infant activity, temperament, and cognition from maternal lactation (Deoni et al. 2013; Hinde and Capitanio 2010). Nursing from different women creates an enriched sensory environment, with social negotiations mediating physiology, resource access, and relationships, processes often difficult to measure in the field (Ellison and Gray 2009). Subtle signals (e.g., slight twisting toward the caregiver, minute movements of mouth and limbs) melded into a seasoned caregiver's intuition and response-especially those who breast-feed — can be easily over- looked by distal or inexperienced observers, making attribution of initiation challenging (Tronick 1989).

Care responds to growth, and Efé infants typically are capable of sitting propped in a lap or supported on the ground by 3.5 months of age and considered sturdy enough to be entertained by others, especially children (Ivey Henry, Morelli, and Tronick 2005). Infant behavior also may be given credit for allonursing cessation, not due to classic attachment per se but to extraordinary selection pressure on infants to stimulate their own mother's milk supply and delay her next ovulation.

\section{Allonursing as Preventative Medicine}

Allonursing provides a unique form of early intervention for Efé mothers and infants. High endemic pathogens of the Congo forest (Gire et al. 2012) impose severe immune costs that should be evident in reproductive and developmental trade-offs (McDade 2012). Efé infant mortality between 14\% and $23 \%$ (Bailey, personal communication; Ivey Henry, unpublished data) is in line or below the hunter-gatherer average of 23\% (Gurven, Kaplan, and Supa 2007), begging the question of the extent to which allonursing and other allocare mitigate significant risks, including the thermoregulatory and hydration challenges of small size. Moreover, microbiotic transfers from allonursing and passing between genetically and microecologically distinct group members may rapidly populate developing immune systems with especially competitive, but locally protective, biota.

Fat and carbohydrates are limited in the Efé environment, but micronutrients obtained dietarily by mothers and transferred through breastmilk also constrain reproduction, development, and immunity (Ramakrishnan et al. 2012; Sirén and Machoa 2008). Iodine deficiency is common in rainforests, severely compromising pregnancy and growth (Zimmermann and Andersson 2012), and pygmies exhibit selection for its uptake (Dormitzer, Ellison, and Bode 1989; Lachance et al. 2012). Iodine is limited in early infancy prior to thyroid maturation, and milk exhibits high daily variation even in well-nourished populations. Moreover, it is poorly stored in maternal tissue (Ahmed et al. 2008; Azizi and Smyth 2009). Efé mothers may buffer threats to their young by sharing lowcost microbiota and indefensible nutrients, smoothing offspring access to immunological and developmental resources. Rather than bank milk, Efé women bank reciprocal investments in young.

\section{Are Humans Selected to Allonurse?}

A common origin of Efé and Aka allonursing is plausible and would be impressive for its persistence. African pygmies share roots at the base of human history with Khoisan and Hadza (Henn et al. 2011; Verdu and Destro-Bisol 2012) but exhibit distinct polymorphisms associated with immune function, metabolism, growth, and skeletal turnover, critical for lacta- 
tion (Campbell and Tishkoff 2010; Mendizabal et al. 2012; Schuh-Huerta and Pera 2011). Less apparent is the phylogenetic, ecological, and cultural diversity of pygmies (Bahuchet 2012; Hewlett 1996), as eastern (e.g., Efé, Mbuti) and western (e.g., Aka, Bofi) populations diverged with fragmentation of Congo forests around 27,000 BP, with little subsequent admixture between them (Batini et al. 2011). As the authors describe, there are substantial differences between Efé and Aka care even in early infancy.

Efé allonursing provides an innovative buffer for mothers and young against the immense odds of their environment. The same impetus may underlie innovations recruiting the milk of other species. Compelling outcomes well motivate inquiry, but a view too far removed from ecological processes that produce them may inform too little, as each trade-off envelops others, tethered less by static defaults than by shared challenges (Clements et al. 2011; Evans et al. 2013). Finergrained analyses are required to hone the confluence of factors favoring allonursing; from these, distal patterns may better emerge.

\section{Acknowledgments}

The research reported here was supported by National Science Foundation grant BNS 8218791, National Institute of Child Health and Development grant RO3-MH38300, and the Spencer Foundation.

\section{Melvin Konner}

Department of Anthropology and Program in Neuroscience and Behavioral Biology, Emory University, Atlanta, Georgia 30322, U.S.A. (antmk@emory.edu). 1 IX 13

Hewlett and Winn's paper sheds valuable light on a hidden subject. We know that wet nurses were commonly used in stratified historical societies, but this was exploitation of lower-ranking women and ironically was often dangerous to the high-born infants, as well as those of the wet nurses who might be deprived (Hrdy 1999). Hewlett and Winn focus on cooperative, not exploitative, practices.

Cooperative breeding was vital to human evolutionary success (Hrdy 2009), so we would expect to see allomaternal nursing, especially in hunter-gatherers. Hewlett and Winn find it in $90 \%$ of cultures, yet it is normative in only $6 \%$, about the same proportion as disallow it. They canvassed scientists who studied infancy in 11 hunter-gatherer cultures; normative allomaternal nursing occurred in five, and it is more likely in tropical forest foragers, but the authors acknowledge that both findings remain conjectural, given the small sample.

Their quantitative analysis of infant observations in two hunter-gatherer groups, the Aka and Efé, led to several important findings. First, even where it is normative, allomaternal nursing accounts for a minority of breast-feeding time
(15\% in the Aka, 23\% in the Efé, who have frequent infertility and more potential allomothers). Second, it is more likely when the mother is off gathering. Third, it is nonexistent or rare by age 1 even where it is normative. Finally, despite the nonrelatives living in hunter-gatherer bands, allomaternal breast-feeders are overwhelmingly genetic relatives of the infant, especially grandmothers.

Hewlett and Winn consider a number of hypotheses relating to these data but find support for only a few. Aside from nourishment, allomaternal nursing should diversify both the microbes the infant is exposed to (with implications for disease as well as the microbiome) and its passive immune protections. But should the positive or negative components win out? There is paleopathological evidence of increased infectious disease after the transition to agriculture, which may have tilted the scales against allomaternal nursing. Fewer births in hunter-gatherers may encourage it. The speculation that tropical foragers have more microbes and therefore need more diverse immune protections is intriguing, but it seems to oppose the hypothesis about non-hunter-gatherers.

The role of maternal work may seem obvious, but the great majority of cultures permit allomaternal nursing in emergencies such as the mother's illness or death; those where it is normative may be more prepared for such emergencies. The fact that most nonmaternal breast-feeders are genetic relatives of the infant adds to much evidence for kin selection in humans, including foragers; cooperative breeding does not recruit just anyone. The data also support the special importance of grandmothers, without a matrilineal-patrilineal difference (Hawkes et al. 1998). Despite the occasional comforting gesture, nursing is not a domain where males can compete; in provisioning other food, they do (Hill and Hurtado 2009; Marlowe 2003, 2010).

Do grandmothers actually supply milk? They certainly can, even if postmenopausal (Slome 1956), and this is clearly of value in some circumstances (Ogunlesi et al. 2008). Nonnursing premenopausal women can also lactate, as can adoptive mothers who have never been pregnant. Given these possibilities, we should perhaps ask why more cultures do not have normative allomaternal nursing rather than why some do.

Attachment is the most likely explanation of why it disappears by a year of age, although teething and increasingly independent immune competence may also be relevant. Despite the Aka being famous for paternal care and the Efé for all allomaternal care, infants in both cultures intensify their relationships with their mothers toward the end of the first year (Konner 2005, 2010). Among the !Kung, who do not have allomaternal nursing, allomaternal care in response to crying is frequent, but mothers remain central (Kruger and Konner 2010). Breast milk aside, parents cannot feed their children without others' help (Howell 2010).

Hewlett and Winn have done a valuable service by starting a systematic discussion of this fascinating aspect of human cooperative breeding. Their paper should stimulate research that will answer the excellent questions they raise about the 
nature and functions of allomaternal nursing. At the same time, it would be interesting to gather data on another hidden subject, maternal and allomaternal "kiss-feeding" (mouth-tomouth transfer of partially chewed foods). This appears to be common cross-culturally (Eibl-Eibesfeldt 1989), may be important for supplemental feeding and weaning, and it raises similar questions about ultimate and proximate causes and consequences regarding infants, mothers, kin groups, nutrition, antigenicity, immunity, and the microbiome.

\section{Daniel Sellen}

Department of Anthropology, University of Toronto, 19 Russell Street, Toronto, Ontario, Canada (dan.sellen@utoronto.ca). 11 X 13

Hewlett and Winn's analysis of the available ethnographic evidence on nonmaternal nursing makes a significant contribution to the anthropology of infant and young child feeding. They offer a well thought-out, conceptual framework for organizing thinking about the social, biological, and economic relationships that structure the forms of allomaternal nursing across populations. Their analysis moves us beyond some historical confusion concerning distinctions between wet nursing, cross-nursing (also called cross-lactation), tandem nursing of biological sibs of different ages, human breast milk exchange, breast milk feeding to adults, cross-species nursing, and animal milk consumption. The effort to draw together firsthand, relatively recent, observational data from forager groups where excellent research teams remain active and eHRAF and other data form the ethnographic literature is a scholarly benefit to anthropologists of all theoretical persuasions. Before this analysis, we were working only with biocultural conceptual frameworks, anecdotal reports of shared nursing, and hypotheses developed in nonhuman evolutionary biology because the practice had drawn little attention from cultural anthropologists. Many anthropologists suspected cross-lactation might be quite common in small-scale societies, and possibly beyond, but we did not have the data organized to test this idea.

Hewlett and Winn are commendably cautious in their conclusions, but to my reading they have discovered clear indications that allomaternal nursing is (i) probably "speciestypical" (documented in a large majority of cultures with data; $>90 \%$ ); (ii) a putative child survival input with potentially large health and developmental benefits to some infants (more than one fifth of breast milk and therefore immune factors and nutrients consumed); (iii) "targeted" in the first half of infancy ("before 4 months," i.e. when breast milk feeding exclusively without other foods is most beneficial to survival and health); and (iv) provided mostly by maternal biological kin (grandmothers being by far the most common alternative providers of breast milk within and between populations, as appears to be the case for other types of child care). It is not trivial that these important exploratory results can be readily integrated into evolutionary theories that humans evolved to rely on a unique type of cooperative breeding and that mothers and others care under complex, context-specific, and changing constraints.

It is more difficult to explain why actual cases of allomaternal nursing appear to be uncommon relative to widespread potential cultural support. Putting aside the acknowledged limitation of unmeasured negative reporting bias, "normative allomaternal nursing” seems surprisingly uncommon given its apparent availability and convenience (occurring as "regular or extensive" in less than half of the foraging groups for which data are collected; 45\%). It is very helpful that Hewlett and Winn have examined the widely held assumptions that soothing infants and helping mothers resolve trade-offs in scheduling work and breast-feeding may be common proximate triggers for allomaternal nursing and are able to suggest that they are often not. Perhaps allomaternal nursing is best understood as a caregiving "option" that is scaffolded into a majority of small-scale societies but mostly drawn upon only in the relatively rare "emergency" instance of maternal death (when it may be crucial); this explanation warrants further empirical investigation.

New evolutionary questions emerge. For example, if indeed allomaternal nursing is not commonly used to replace colostrum feeding of newborns, it may yet prove to be a common solution to the problem of soothing hungry neonates in the hours between parturition and milk letdown (lactogenesis stage 2). The practice of hand expressing and discarding colostrum and first milk remains a puzzle for evolutionary anthropologists, given the known benefits of timely initiation of breast-feeding; it may be analogous to early umbilical cord clamping after parturition, now linked to iatrogenic iron deficiency infants. Although the biomedical literature indicates discarding of colostrum may be widespread in many large contemporary populations, was it really very common in small-scale societies? These interesting results add urgency to calls for more standardized comparisons of infant care practices.

This careful analysis of a mix of data of varying quality and granularity narrows a critical knowledge gap and indirectly provides some tentative answers to questions of direct relevance to frameworks and strategies for protecting, promoting, and supporting breast-feeding globally: Are women who practice or accept ideas about cross-nursing best construed as pathbreaking, pathological, or plain practical? Are sisters, mothers, friends or employees common or preferred allomaternal nurses? Do the social relations of allomaternal nursing offer precedents for organizing modern human milk banking and distribution systems? Such questions are often asked of applied anthropologists by health practitioners and policy makers, particularly those involved with prevention of maternal to child transmission of infectious diseases such as HIV. Now we can say much more to health practitioners about the cross-cultural distribution of allomaternal nursing and 
therefore the potential for benefit and risk to various human health outcomes.

\section{Akira Takada \\ Center for African Area Studies (CAAS), Graduate School of Asian and African Area Studies (ASAFAS), Kyoto University, 46 Yoshida-Shimoadachi, Sakyo, Kyoto, 606-8501, Japan (takada@jambo.africa.kyoto-u.ac.jp).22 VIII 13}

Hewlett and Winn's article is a fine pioneering work on nonmaternal breast-feeding, which "is common cross-culturally but is poorly understood and no systematic studies exist." As a fellow researcher studying child rearing in hunter-gather populations, I was excited to read the article. It will be a great contribution to the study of child rearing and to the broader domains of anthropology, particularly for the following qualities.

1. The methods adopted in this article are of particular interest. This article uses focal follow observations of Aka, Efé, and Ngandu infants, semistructured interviews with Aka mothers, ethnographic reports from selected researchers working with hunter-gatherers, and a systematic survey of numerous eHRAF cultures. Each method has limitations that represent teething problems for pioneering research. When these methods are combined, however, they make up for one another's limitations. For instance, although information on allomaternal nursing in populations other than Aka is more or less limited, the existing information makes it possible to locate Aka allomaternal nursing practices on a map of those other populations. Meanwhile, focal follow observations of Aka, Efé, and Ngandu infants help us reconsider discrepancies between practices and interview data on this issue.

2. This study is important in that it illuminates a distinction between early and late infancy regarding nursing practices. The authors clarified that allomaternal nursing in the Aka and Efé disappears by the time infants are 12 months old, even though Aka and Efé infants continue to be breast-fed until ages 3 and 2, respectively. This suggests that nursing practices have different or multiple functions depending on the age of children, and the functions of nonmaternal nursing only partially overlap with those of maternal nursing. They then asserted that infant preference for nursing from a specific caregiver (i.e., mother) played a role in the decline of allomaternal nursing. This is a reasonable interpretation and can be further developed in relation to point 4 below.

As the authors mentioned, this study is exploratory and raises more questions than it answers. It will be particularly important to promote further examination of the following two points.

3. The authors discussed various evolutionary hypotheses. It appears that, although allomaternal nursing could have an impact on reproductive fitness, there are several important factors that were not considered in this paper. For example, the presence or absence of infanticide and swaddling may function as mediating factors in establishing the pattern of maternal and nonmaternal nursing. Additionally, nursing practices would be related to the availability of agricultural and livestock products (e.g., grain, animal milk) that could be used as baby solids. An increasing number of studies have demonstrated multifaceted historical interplay between hunter-gathers and agro-pastoralists, and a number of huntergatherer populations have had access to these products. If these factors are taken into consideration, the finding that "allomaternal nursing is more common among foragers living in tropical forest rather than arid environments[, but] Ngandu farmers, who live in the same tropical forest as do Aka foragers, strongly discourage allomaternal nursing" might be better understood.

4. My personal viewpoint on this issue is close to the combination of cultural models and stressful infant hypotheses. I have worked among various groups of San, who widely believe that mothers' milk belongs to the last born; when mothers become pregnant, they stop nursing the preceding child. Allomaternal nursing is normally not allowed. Mothers nurse their infants briefly and frequently, with short intervals between nursing periods (Konner and Worthman 1980; Takada 2005). The recurrent nursing results in a small amount of milk being consumed per nursing bout. Instead, mothers often contingently accommodate nursing to infant movement, including fretful behaviors. Other caregiving behaviors (e.g., "gymnastic" behavior) also contribute to shaping the sequential organization of interactions (Takada 2005, 2011). Thus, cultural models and infant movements are used as resources in creating the distinctive nursing pattern of the San.

The authors claimed that the stressed infant hypothesis did not find much support because "infants who received allomaternal nursing did not fuss or cry any more frequently than other infants." However, mothers and allomothers would often start nursing before an infant began to fuss or cry because they quickly noticed the infant's minute movements. Detailed microanalysis of nursing episodes will clarify the participation framework of interactions in which maternal and nonmaternal nursing occur. The hypothesis should be examined in light of the whole participation framework.

The mother-child relationship is supported by a wider sociocultural network, and allomaternal nursing is interwoven with other forms of allomothering. It is necessary to further scrutinize how the forms of allomothering are given shape in order to "explain the enormous within and between cultural diversity in allomaternal nursing."

\section{Hideaki Terashima}

Faculty of Humanities and Sciences, Kobe Gakuin University, 518 Arise, Ikawadani-cho, Nishi-ku, Kobe-shi, 651-2180, Japan (terashima@kh.rim.or.jp).26 VIII 13

Barry S. Hewlett and Steve Winn's paper “Allomaternal Nursing in Humans" provides important quantitative behavioral 
data and insights based on intensive fieldwork and a crosscultural survey on a particular nursing method called "allomaternal breast-feeding," which has been observed in many human societies, especially hunter-gatherers. However, although allomaternal care for infants has been so commonly observed in every human society, surprisingly little substantial data has been collected about allomaternal breast-feeding behavior.

The saying that "you see what you want to see" certainly seems to be applicable to allomaternal breast-feeding. I conducted fieldwork among Efé Pygmy hunter-gatherers from the late 1970s to the middle of the 1980s and saw juveniles, adolescents, fathers, grandmothers, or adult women other than the mothers in the hunting camps taking care of young infants every day. However, I never observed or, more probably, did not notice allomaternal breast-feeding at all, although the Efé Pygmies are said in this paper to be one of the hunter-gatherer groups who accept allomaternal breastfeeding as a normal behavior.

As Hewlett and Winn suggest, the reason that allomaternal breast-feeding did not get much attention from fieldworkers including myself may be ascribed to the behavioral characteristics of allomaternal breast-feeding. First, the behavior is observable when the infant is within a relatively short age range, beginning from very early months until he or she is around 4 months old and disappearing completely after he or she is a year old. Second, the shortness of the each bout of nursing may make observation difficult. The Aka Pygmies breast-feed 4.0 times per hour with a duration of 2.4 minutes each time (Hewlett et al. 2000). Ayako Hirasawa (2005) reported that the Baka Pygmies of southeastern Cameroon breast-feed more frequently and briefly at 4.8 times per hour with each breast-feeding lasting 1.3 minutes. The focal subject sampling technique that the authors used to record the behavior of each focal person played a big role in showing inconspicuous allomaternal breast-feeding clearly. Moreover, it may be difficult to imagine that grandmothers could nurse the babies.

Interestingly, allomaternal breast-feeding was not rare historically among human populations, but it was mostly limited to special situations such as when the mother could not breast-feed as a consequence of disease or death after giving birth, or where a social custom like wet nursing by lowerclass women was prevailing. So, the discovery of normative allomaternal breast-feeding in extant hunting and gathering societies brings about intriguing biological, ecological, and cultural questions about its value and implications for human evolution.

The authors grapple with these questions by exploring worldwide ethnographic data and various hypotheses on the reasons for allomaternal breast-feeding behavior, including that of nonhuman animals. They found that environmental factors may partly explain the behavior because there are clear differences in allomaternal breast-feeding between huntergatherers who live in wet environments such as tropical forests and those who live in drier environments such as the savanna or woodlands. Normative allomaternal breast-feeding is carried out by the hunter-gatherers in the former environment but not in the latter. The authors also note that allomaternal breast-feeding is conducted mainly by women related to the infants-especially grandmothers - which indicates the possibility that allomaternal nursing is related to the enhancement of inclusive fitness. This may also be related to the "grandmother hypothesis" in human evolution.

However, as the authors mention, there are large intracultural and intercultural variations and nondeterministic conclusions that could be drawn for the present. More studies are expected on this rather mysterious allomaternal breastfeeding behavior to understand its value and implications in the hunting and gathering life and the complicated interaction between nature and culture in human evolution.

\section{Reply}

We sincerely thank the commentators for their insightful critiques, new data, supportive and encouraging comments, and ideas for future research. We particularly enjoyed the new ethnographic data on allomaternal nursing and were delighted that anthropologists engaged in public health policy, such as Sellen, Ivey Henry, Morelli, and Tronick, found the paper useful. It was also gratifying that none of the commentators challenged the empirical results-for example, the cultures in which it exists, the narrow age range in which it occurs, who provides allomaternal nursing, and so forth. Several commentators did question our interpretations of the data and identify limitations of our research methods.

\section{Future Field Research}

Most of the critical comments dealt with the limitations of our data and suggest ways to improve future systematic studies of allomaternal nursing. Below we summarize their theoretical and methodological suggestions that a graduate student or anyone interested in future research on allomaternal nursing could use in a research proposal.

Conduct detailed behavioral observations during and interview mothers about the neonatal period. Crittenden, Sellen, Ivey Henry, Morelli, and Tronick identify this limitation in our study. As mentioned in the paper, our field studies utilized behavioral observations to try to understand the daily lives of forager infants. Allomaternal nursing was not the focus of our research, and consequently we did not target the neonatal period. This period is critical for allomaternal nursing because studies in the West indicate that women are most likely to turn to supplementation at this time, women in small-scale cultures may practice colostrum taboos during this period, 
the frequency of infant crying is high at this time, and milk production may be low.

Videotape mother and allomother nursing bouts. Takada points out that some of our results may be misleading, such as lack of evidence for the fussy infant hypothesis, because our behavioral observations were not sensitive enough to capture particular interactions. He suggests using microanalysis of videos of nursing mothers to determine whether mothers or allomothers were able to read subtle infant movements before they started to fuss or cry that led them to nurse. Microanalysis of videotapes could be used to compare a broad range of subtle mother/allomother-infant interactions (McKenna et al. 1994).

Interview allomothers. We interviewed mothers, but Fouts explains that it is also essential to interview allomothers to understand their motivations for nursing infants who are not their offspring. She provides a few examples of how interviews with Bofi forager allomaternal females provide insights into theoretical issues about why allomothers nurse infants.

Measure infant's milk intake. We used behavioral observations of infants sucking on the breast to estimate the time allomothers breast-feed their infants, but we did not directly measure milk intake. This is essential to evaluate several of the evolutionary hypotheses. Hinde, Crittenden, Terashima, and other researchers (Miller et al. 2013) question the usefulness of behavioral observations to estimate milk intake. This is a valid critique, but unfortunately we are unaware of simple and nonevasive methods to measure milk intake. The methods used with captive nonhuman animals or in laboratory contexts, for example, weighing of mother and infant before and after feeding and other methods described in Miller et al. (2013) are not feasible in small-scale cultures, especially in forager cultures where mothers nurse about four times an hour. We are confident researchers can develop nonevasive, ethical, and sensitive methods for future studies.

Conduct biological analysis of breastmilk of mothers and allomothers. Hinde points out that breastmilk contains hundreds of bioactive constituents that vary among mothers and allomothers. Several random sampling techniques exist and could be used in small-scale culture field settings (Miller et al. 2013). Hinde indicates that mismatches may exist between mother's and allomother's milk but that the allomother's milk may provide immune benefits. We know very little at this point. It is worth noting again that variability in the quality of mother's milk is recognized by Aka mothers; they pay keen attention to the foods consumed by women who allonurse their infants.

\section{Questions and Alternative Hypotheses}

Ivey Henry, Morelli, and Tronick ask several important questions not addressed in our paper and offer alternative hypotheses to the ones we propose to explain some of the variability. We list their questions and describe what we know from our data. First, "Is allonursing an additive or replace- ment resource for maternal milk?" In instances when the mother is sick or dies, it is clearly replacement, but the fact that it usually occurs within this limited age range suggests it may be additive. Among the Aka, no significant difference existed between infants who received allomaternal nursing and those who did not in the total minutes the infants were breast-fed. Mothers who did not receive allomaternal assistance nursed their infants about $17 \%$ more often on average than mothers who received assistance. This suggests that allomaternal nursing replaces milk not received from mother and demonstrates the potential substantial energy savings a mother may receive from an allomother.

"Is allomaternal nursing performed within the continuous flow of specialized handling that youngest infants require, or do mothers specifically pass their infants to allonurse?" We do not have systematic data to answer the question, but our paper suggests it is within the context of daily care. Statistical analysis in the paper indicates that Aka allomothers most likely to nurse a young infant were those who spent the greatest amount of time holding the infant. Aka mothers may pass a fussy infant who does not want to nurse with her to another woman; she may, in turn, nurse the infant. Among the Efé, informal observations indicate it is most likely to occur when the mother is out collecting firewood or water and another woman holding the infant nurses the baby. We do not have evidence that mothers intentionally pass their infants to another woman to nurse, but if the mother is not around and a young girl or man is holding the baby and it starts to fuss, it is often passed to someone who can nurse it. In-depth interviews with mothers with young infants would help better understand the rationale and contexts of transfers from mothers to allomothers.

The commentators ask, to what extent do lactating mothers allonurse? This is another good question that we are unable to systematically answer with our data. The question is proposed, in part, because Aka grandmothers often allonurse, and commentators want to know the lactation status of the grandmother. We have observed allomaternal nursing in all three contexts they describe-grandmothers nursing their own child and grandchild, grandmothers without young children who have relactated and nurse, and very old grandmothers without any fluid but who put the infants to their breasts to soothe them. The second context was the most common. By contrast to the Aka, Efé allomaternal nursing by grandmothers is rare. It is worth noting that among the Efé, a newborn baby is passed to each woman and girl in the birth home regardless of lactational status. An allomother nurses the newborn a few times a day until the mother's milk comes in, and if no lactating women live in the camp, a woman from a nearby camp is recruited (Morelli and Winn 1987).

Ivey Henry, Morelli, and Tronick also offer an alternative hypothesis to explain why infants decrease allomaternal nursing in late infancy. They hypothesize that infants prefer mother not because of the attachment process that we and 
Konner suggest in late infancy but to stimulate their own mother's milk supply and delay her next ovulation.

Reciprocity is a potential explanatory factor for allomaternal nursing, and Ivey Henry, Morelli, and Tronick suggest that reciprocity feedback may be a maternal strategy. We did not find evidence for this among the Aka or Efé, and our observations suggest it is more of a "it takes a village" or "giving environment" (Bird-David 1990) cultural value rather than "you scratch my back." Fouts's commentary on interviews with allomothers also indicates that reciprocity is not important for long-term allomaternal nursing. But as described in the paper, evidence for reciprocity exists in other cultures, such as Manus and several milk-kinship cultures.

In terms of explaining cross-cultural variability, Konner hypothesizes that allomaternal nursing may be more frequent among foragers than among farmers. He suggests that allomaternal nursing may have declined with domestication because of increases in infectious and parasitic diseases associated with sedentism and higher population density. Agricultural women are predicted to have a greater concern about maternal-infant transmission of infectious disease. It is a hypothesis worth testing, but our limited eHRAF study does not support the hypothesis, as only one of the six cultures with normative allomaternal nursing was a forager group. Takada also suggests that future cross-cultural studies should consider factors such as presence or absence of infanticide, swaddling, and the availability of agricultural and livestock products (e.g., grain, animal milk) that could be used as baby solids.

\section{Frequency of Allomaternal Nursing}

It is our impression that the frequency of allomaternal nursing has been underestimated in the ethnographic record. Sellen, Konner, and others ask why normative allomaternal nursing is not more common in hunter-gatherers and in the general cross-cultural record. It is a good question, and we tried to offer ecological and cultural factors that influence why a society does or does not practice allomaternal nursing, but as most of the commentators point out, we need substantially more data. One issue worth mentioning here is documentation in the cross-cultural record. Terashima provides an honest and instructive commentary when he reports that he never saw allomaternal nursing even though it is particularly common among the Efé. His research did not focus on infants, he did not conduct focal follows of women, and Efé camps usually have only one or two infants due to small camp sizes. Research methods and settings along with our findings that allomaternal nursing occurs within a relatively narrow infant age range and that forager nursing bouts are often of short duration contribute to the difficulty in observing the practice. When Tronick, Morelli, and Winn (1987) published their work on Efé allomaternal nursing, at first I (Hewlett) did not think it occurred often with Aka because I was working primarily with fathers and older infants; it was not until I started to conduct behavioral observations with a large sample of younger infants (Hewlett et al. 1998) that I realized it was so common. Recently I presented allomaternal nursing data at a professional meeting, and prominent male and female Congo Basin ethnographers working with Aka and other hunter-gatherers responded the same way as Terashima did with the Efé; that is, it was a surprise to them that it existed. The short and early infant age range within which it usually takes place, the briefness of nursing bouts, and the fact that most anthropologists come from cultures with mother-only nursing likely limit its cross-cultural documentation. It is most likely to be noted ethnographically when a healthy middle-aged woman dies of an illness or in childbirth (traumatic public events observable to an anthropologist) and another woman starts to allonurse the baby so it will survive. It takes public events to be documented.

\section{The Limits of Allomaternal Nursing}

Ivey Henry, Morelli, and Tronick share a sad story of a mother who died in childbirth and the father could not convince another woman to nurse the newborn, so the baby died. The story shocked both of us. We have both observed infants with congenital (hydrocephalic) or developmental issues being allonursed and cared for by several people. It is difficult for us to imagine the situation among the Aka or Efé and sense that there is more to the story, but it illustrates several issues. First, and as Ivey Henry, Morelli, and Tronick emphasize, pronounced intracultural diversity exists. While shocking to us, it illustrates that in natural fertility populations, not every child is treated the same way.

The story also identifies an issue not developed in our paper-short-term versus long-term investment in allomaternal nursing. Allomaternal breast-feeding while mother is away for a few hours gathering or collecting firewood is immensely different and of substantially lower cost than taking over primary breast-feeding when a mother dies or is sick for a long time.

We generally agree with Hinde that "allomaternal nursing may have importantly contributed to infant development and well-being, but little empirical data at present allow for such a conclusion." On the other hand, cases reported by Fouts and Ivey Henry, Morelli, and Tronick demonstrate that some infants die if they do not receive allomaternal nursing and that at least some forager females feel that their allonursing influences infant survival. Peter Gardner (personal communication) conducted research with Paliyan foragers of South India and indicates that Paliyan absolutely do not allonurse and recounted a story of a mother with a large tropical ulcer on her breast that made it impossible for her to nurse her infant. The infant died.

We agree with Hinde that the nutritional value of allonursing is uncertain, but to characterize the amounts of allomaternal nursing as "relatively limited" is questionable. Some Aka and Efé infants received $50 \%$ or more of their nursing 
from allomothers, and the mean for 1-2-month-old Efé infants who received allomaternal nursing was $28 \%$ of total breast-feeding time. One has to remember that the mean percentages are based upon only 4 hours of observation per infant among the Efé and 9 hours of observation per infant among the Aka. Also, as mentioned above, Aka mothers who receive nursing assistance from allomothers may reduce their breast-feeding time and presumably costs of lactation by an average of $17 \%$.

\section{Multiple Roles of Allomaternal Nursing}

As with breast-feeding in general (Fouts, Hewlett, and Lamb 2012), allomaternal nursing is not only about nutrition. The interactions transmit information about the nature of socialcultural-emotional relations. The nutritional value of allomaternal nursing may be uncertain, especially from grandmothers, but, as Hinde notes, allomaternal milk, even at lower nutritional value, can signal milk security. The secure nursing environment is consistent with several other cultural childcare practices and values among the Aka and Efé (Hewlett, forthcoming).

Fouts provides an example of how cultural models influence allomaternal nursing; a Bofi grandmother thought her daughter's early weaning would lead to the death of her grandchild so she decided to allonurse. Fouts also suggests that Bofi forager cultural norms promote extensive giving to others and that allomaternal nursing is a specific extension of these norms.

Hinde's comment on allomaternal nursing among the Himba also suggests that cultural models play a role-sisters and grandmothers provide allomaternal nursing even though milk is readily available from cows and goats. We look forward to more details from Scelza and Hinde on allomaternal nursing in these groups.

\section{Final Comments}

Ivey Henry, Morelli, and Tronick question the use of the term "normative" for cultures in which over $90 \%$ of infants receive allomaternal nursing because it obscures intracultural diversity. We are, of course, interested in both intercultural and intracultural diversity and are open to alternative terms. Suggestions?

Konner describes an interesting form of infant feeding among the !Kung, called kiss-feeding, and suggests it might provide insights into allomaternal feeding and weaning practices. Biological fluids are exchanged and some costs are involved for the provider. It is a type of infant feeding in which fathers and other males could participate. Hewlett observed it several times among the Aka (mostly by fathers), but according to Winn it was not observed during any of his formal or informal observations of Efé infants. Questions we asked about allomaternal nursing would be similar for kiss-feed- ing - how often does it occur, who provides it, and in what contexts does it take place?

Finally, an aim of the paper was to make allomaternal nursing not so "mysterious" (Tereshima) or viewed as "pathological" (Sellen). It is a human universal in that it is known and practiced at some level in over $90 \%$ of the world's cultures, but its frequency and contexts vary enormously within and between cultures because of an array of ecological, cultural, biological, and developmental factors. As several of the commentators emphasize, many mysteries remain to be discovered and understood, and research methods need to be improved. Our research focused on small-scale cultures, and studies with these cultures are urgently needed because the practice is rapidly declining with exposure to "modernization" and Western-based ideas about infant health and development. But research is also needed in developed countries where the need for breastmilk is increasing and a vast array of cyber networks and formal and informal milk exchanges have emerged.

-Barry S. Hewlett and Steve Winn

\section{References Cited}

Adriani, N. 1951. The Bare'e-speaking Toradja of central Celebes (the East Toradja). Amsterdam: Noord-Hollandsche Uitgevers Maatschappii.

Ahmed, Osama M., A. W. el-Gareib, A. M. el-Bakry, S. M. Abd el-Tawab, and R. G. Ahmed. 2008. Thyroid hormones states and brain development interactions. International Journal of Developmental Neuroscience 26(2):147209. doi:10.1016/j.ijdevneu.2007.09.011. [PIH/GAM/ET]

Alexander, R. D. 1974. The evolution of social behavior. Annual Review of Ecology and Systematics 5:325-383.

American Academy of Pediatrics. 2005. Breastfeeding and the use of human milk. Pediatrics 115:496-506.

Auerbach, Kathleen G., and J. L. Avery. 1981. Induced lactation: a study of adoptive nursing by 240 women. American Journal of Diseases of Children 135:340-344. [KH]

Azizi, Fereidoun, and Peter Smyth. 2009. Breastfeeding and maternal and infant iodine nutrition. Clinical Endocrinology 70(5):803-809. doi:10.1111/ j.1365-2265.2008.03442.x. [PIH/GAM/ET]

Bahuchet, Serge. 2012. Changing language, remaining Pygmy. Human Biology 84(1):11-43. doi:10.3378/027.084.0101. [PIH/GAM/ET]

Bailey, Robert C., and Irven DeVore. 1989. Research on the Efé and Lese populations of the Ituri Forest, Zaire. American Journal of Physical Anthropology 78:459-471. [HNF, PIH/GAM/ET]

Bailey, Robert C., and Nadine R. Peacock. 1988. Efé pygmies of northeast Zaire: subsistence strategies in the Ituri Forest. In Coping with uncertainty in food supply. I. de Garine and G. A. Harrison, eds. Pp. 88-117. Oxford: Oxford University Press. [PIH/GAM/ET]

Baldovino, M. C., and M. S. Di Bitetti. 2008. Allonursing in tufted capuchin monkeys (Cebus nigrtus): milk or pacifier? Folia Primatology 79:79-92.

Barr, R. G., M. Konner, R. Bakeman, and L. Adamson. 1991. Crying in !Dung San infants: a test of the cultural specificity hypothesis. Developmental Medicine and Child Neurology 33(7):601-610. doi:10.1111/j.1469-8749.1991 .tb14930.x. [PIH/GAM/ET]

Basden, G. T. 1966. Among the Ibos of Nigeria: an account of the curious and interesting habits, customs and beliefs of a little known African people by one who has for many years lived amongst them on close and intimate terms. London: Cass.

Batini, Chiara, Joao Lopes, Doron M. Behar, Francesc Calafell, Lynn B. Jorde, Lolke Van der Veen, Lluís Quintana-Murci, Gabriella Spedini, Giovanni Destro-Bisol, and David Comas. 2011. Insights into the demographic history of African Pygmies from complete mitochondrial genomes. Molecular Biology and Evolution 28(2):1099-1110. doi:10.1093/molbev/msq294. [PIH/ GAM/ET] 
Bird-David, N. 1990. The giving environment: another perspective on the economic system of gatherer-hunters. Current Anthropology 31:183-196.

Boyd, R., and P. J. Richerson. 2005. The origin and evolution of cultures. New York: Oxford University Press. [HNF]

Briga, M., I. Pen, and J. Wright. 2012. Care for kin: within-group relatedness and allomaternal care are positively correlated and conserved throughout the mammalian phylogeny. Biology Letters 8(4):533-536. doi:10.1098/ rsbl.2012.0159. [PIH/GAM/ET]

Cameron, E. Z. 1998. Is suckling behaviour a useful predictor of milk intake? A review. Animal Behavior 56:521-532.

Campbell, Michael C., and Sarah A. Tishkoff. 2010. The evolution of human genetic and phenotypic variation in Africa. Current Biology 20(4):R166R173. doi:10.1016/j.cub.2009.11.050. [PIH/GAM/ET]

Champagne, Frances A. 2011. Maternal imprints and the origins of variation. Hormones and Behavior 60(1):4-11. doi:10.1016/j.yhbeh.2011.02.016. [PIH/ GAM/ET]

Clements, Michelle N., Tim H. Clutton-Brock, Fiona E. Guinness, Josephine M. Pemberton, and Loeske E. B. Kruuk. 2011. Variances and covariances of phenological traits in a wild mammal population. Evolution 65(3):788801. doi:10.1111/j.1558-5646.2010.01161.x. [PIH/GAM/ET]

Cowett, Richard M., and Hussien M. Farrag. 2004. Selected principles of perinatal-neonatal glucose metabolism. Seminars in Neonatology 9(1):3747. doi:10.1016/S1084-2756(03)00113-1. [PIH/GAM/ET]

Creel, S. R., S. L. Montfort, D. E. Wildt, and P. M. Waser. 1991. Spontaneous lactation is an adaptive result of pseudopregnancy. Nature 351:660-662.

Culwick, A. T. 1935. Ubena of the rivers. London: Allen \& Unwin.

Daly, M., and M. Weghorst. 1982. Male sexual jealousy. Ethology and Sociobiology 3:11-27.

DaMota, K., J. Bañuelos, J. Goldbronn, L. E. Vera-Beccera, and M. J. Heinig. 2012. Maternal request for in-hospital supplementation of healthy breastfed infants among low-income women. Journal of Human Lactation 28:476482. [ANC]

Deoni, Sean C. L., Douglas C. Dean III, Irene Piryatinksy, Jonathan O’Muircheartaigh, Nicole Waskiewicz, Katie Lehman, Michelle Han, and Holly Dirks. 2013. Breastfeeding and early white matter development: a crosssectional study. NeuroImage 82: 1-10. doi:10.1016/j.neuroimage.2013.05 .090. [PIH/GAM/ET]

Dettwyler, Katherine A., and Claudia Fishman. 1992. Infant feeding practices and growth. Annual Review of Anthropology 21:171-204. doi:10.1146 /annurev.an.21.100192.001131. [PIH/GAM/ET]

Dietz, William H., Bruno Marino, Nadine R. Peacock, and Robert C. Bailey. 1989. Nutritional status of Efe pygmies and Lese horticulturists. American Journal of Physical Anthropology 78(4):509-518. doi:10.1002/ajpa.133078 0406. [PIH/GAM/ET]

Dormitzer, P. R., P. T. Ellison, and H. H. Bode. 1989. Anomalously low endemic goiter prevalence among Efé pygmies. American Journal of Physical Anthropology 78(4):527-531. doi:10.1002/ajpa.1330780408. [PIH/GAM/ET]

Douglas, Pamela S., and Peter S. Hill. 2011. The crying baby: what approach? Current Opinion in Pediatrics 23(5):523-529. doi:10.1097/MOP.0b013e32834 alb78. [PIH/GAM/ET]

Dunn, F. L. 1968. Epidemiological factors: health and disease in hunter-gatherers. In Man the hunter. R. B. Lee, ed. Pp. 221-228. Chicago: Aldine.

Dunsworth, Holly M., Anna G. Warrener, Terrence Deacon, Peter T. Ellison, and Herman Pontzer. 2012. Metabolic hypothesis for human altriciality. Proceedings of the National Academy of Sciences 109(38):15212-15216. doi: 10.1073/pnas.1205282109. [PIH/GAM/ET]

Durham, W. 1992. Coevolution: genes, culture and human diversity. Stanford, CA: Stanford University Press.

Eberle, M., and P. M. Kappeler. 2006. Family insurance: kin selection and cooperative breeding in a solitary primate (Microcebus murinus). Behavioral Ecology and Sociobiology 60:582-588.

Eibl-Eibesfeldt, I. 1989. Human ethology. New York: Aldine de Gruyter. [MK]

Ellison, Peter T. 2001. On fertile ground. Cambridge, MA: Harvard University Press. [PIH/GAM/ET]

Ellison, Peter T., and Peter B. Gray. 2009. Endocrinology of social relationships. Cambridge, MA: Harvard University Press. [PIH/GAM/ET]

Evans, Matthew R., Volker Grimm, Karin Johst, Tarja Knuuttila, Rogier de Langhe, Catherine M. Lessells, Martina Merz, Maureen A. O’Malley, Steve H. Orzack, Michael Weisberg, Darren J. Wilkinson, Olaf Wolkenhauer, and Tim G. Benton. 2013. Do simple models lead to generality in ecology? Trends in Ecology \& Evolution 28(10):578-583. doi:10.1016/j.tree.2013.05.022 [PIH/ GAM/ET]
Field, C. J. 2005. The immunological components of human milk and their effect on immune development in infants. Journal of Nutrition 135:1-4.

Fildes, V. 1988. Wet nursing: a history from antiquity to the present. Oxford: Basil Blackwell.

Flinn, Mark V., Pablo A. Nepomnaschy, Michael P. Muehlenbein, and Davide D. Ponzi. 2011. Evolutionary functions of early social modulation of hypothalamic-pituitary-adrenal axis development in humans. Neuroscience and Biobehavioral Reviews 35(7):1611-1629. doi:10.1016/j.neubiorev.2011.01.005. [PIH/GAM/ET]

Fouts, Hillary N., and Robyn A. Brookshire. 2009. Who feeds children? A child's-eye-view of caregiver feeding patterns among the Aka foragers in Congo. Social Science and Medicine 69(2):285-292. doi:10.1016/j.socscimed .2009.05.016. [PIH/GAM/ET]

Fouts, Hillary N., B. S. Hewlett, and M. E. Lamb. 2012. A biocultural approach to breastfeeding interactions in central Africa. American Anthropologist 114: 123-136.

Funkhouser, Lisa J., and Seth R. Bordenstein. 2013. Mom knows best: the universality of maternal microbial transmission. PLoS Biology 11(8): e1001631. [KH]

Gero, S., D. Engelhaupt, L. Rendell, and H. Whitehead. 2009. Who cares? Between-group variation in alloparental caregiving in sperm whales. Behavioral Ecology and Sociobiology 38:237-244.

Gindler, J., M. U. Nwankwo, J. A. Omene, R. H. Glew, and I. M. Roberts. 1985. The quality of milk in a 65 year-old Nigerian woman. Nutrition Research 5(11):1209-1213. [KH]

Gire, Stephen K., Matthew Stremlau, Kristian G. Andersen, Stephen F. Schaffner, Each Bjornson, Kathleen Rubins, Lisa Hensley, Joseph B. McCormick, Eric S. Lander, Robert F. Garry, Christian Happi, and Pardis C. Sabeti. 2012. Emerging disease or diagnosis? Science 338(6108)(8):750-752. doi:10.1126/ science.1225893. [PIH/GAM/ET]

Gorer, G. 1938. Himalayan village: an account of the Lepchas of Sikkim. London: Michael Joseph Ltd.

Gottlieb, A. 2004. The afterlife is where we come from: the culture of infancy in West Africa. Chicago: University of Chicago Press.

Gurven, Michael, Hillard Kaplan, and Alfredo Zelada Supa. 2007. Mortality experience of Tsimane Amerindians of Bolivia: regional variation and temporal trends. American Journal of Human Biology 19(3):376-398. doi: 10.1002/ajhb.20600. [PIH/GAM/ET]

Hatchwell, Ben J., and Jan Komdeur. 2000. Ecological constraints, life history traits and the evolution of cooperative breeding. Animal Behaviour 59(6): 1079-1086. doi:10.1006/anbe.2000.1394. [PIH/GAM/ET]

Hawkes, K., J. F. O'Connell, N. G. Jones, H. Alvarez, and E. L. Charnov. 1998. Grandmothering, menopause, and the evolution of human life histories. Proceedings of the National Academy of Sciences 95:1336.

Heinrich, J., and R. Boyd. 1998. The evolution of conformist transmission and the emergence of between-group differences. Evolution and Human Behavior 19:215-241. [HNF]

Henn, Brenna M., Christopher R. Gignoux, Matthew Jobin, Julie M. Granka, J. M. Macpherson, Jeffrey M. Kidd, and Laura Rodríguez-Botigué, et al. 2011. Hunter-gatherer genomic diversity suggests a Southern African origin for modern humans. Proceedings of the National Academy of Sciences 108(13):5154-5162. doi:10.1073/pnas.1017511108. [PIH/GAM/ET]

Hewlett, B. S. 1991a. Demography and childcare in preindustrial societies. Journal of Anthropological Research 47:1-37.

- $1991 \mathrm{~b}$. Intimate fathers: the nature and context of Aka Pygmy paternal infant care. Ann Arbor: University of Michigan Press. [HNF]

- 1996. Cultural diversity among African Pygmies. In Cultural diversity among twentieth-century foragers: an African perspective. S. Kent, ed. Pp. 215-244. Cambridge: Cambridge University Press.

- Forthcoming. Hunter-gatherer childhoods in the Congo Basin. In Hunter-gatherers of the Congo Basin: cultures, histories, and biology of African Pygmies. Piscataway, NJ: Transaction.

Hewlett, B. S., H. N. Fouts, A. H. Boyette, and B. L. Hewlett. 2011. Social learning among Congo Basin hunter-gatherers. Philosophical Transactions of the Royal Society B 366:1168-1178.

Hewlett, B. S., M. E. Lamb, B. Leyendecker, and A. Schölmerich. 2000. Internal working models, trust, and sharing among foragers. Current Anthropology 41:287-297.

Hewlett, B. S., M. E. Lamb, D. Shannon, B. Leyendecker, and A. Schölmerich. 1998. Culture and early infancy among Central African foragers and farmers. Developmental Psychology 34:653-661.

Hewlett, B. S., J. M. H. van de Koppel, and M. van de Koppel. 1986. Causes 
of death among Aka Pygmies of the Central African Republic. In African Pygmies. L. L. Cavalli-Sforza, ed. Pp. 45-63. New York: Academic Press.

Hill, K., and A. M. Hurtado. 2009. Cooperative breeding in South American hunter-gatherers. Proceedings of the Royal Society B-Biological Sciences 276(1674): 3863-3870. [MK]

Hill, K. R., R. Walker, M. Mozicevic, J. Eder, T. Headland, B. Hewlett, A. M. Hurtado, F. Marlowe, P. Wiessner, and B. Wood. 2011. Coresidence patterns in hunter-gatherer societies show unique human social structure. Science 331:1286-1289.

Hinde, Katie. 2013. Lactational programming of infant behavioral phenotype. In Building babies: primate development in proximate and ultimate perspective. Kathryn B. H. Clancy, Katie Hinde, and Julienne N. Rutherford, eds. Pp 281-302. New York: Springer. [KH]

Hinde, Katie, and John P. Capitanio. 2010. Lactational programming? Mother's milk energy predicts infant behavior and temperament in Rhesus macaques (Macaca Mulatta). American Journal of Primatology 72:522-529. doi: 10.1002/ajp.20806. [PIH/GAM/ET]

Hinde, Katie, and Lauren M. Milligan. 2011. Primate milk synthesis: proximate mechanisms and ultimate perspectives. Evolutionary Anthropology 20:9-23. [ANC, KH, PIH/GAM/ET]

Hirasawa, Ayako. 2005. Infant care among the sedentarized Baka huntergatherers in southeastern Cameroon. In Hunter-gatherer childhoods: evolutionary, developmental and cultural perspectives. B. S. Hewlett and M. E. Lamb, eds. Pp. 365-384. New Brunswick, NJ: Aldine Transaction.

Holmes, A. V. 2013. Establishing successful breastfeeding in the newborn period. Pediatric clinics of North America 60:147-168. [ANC]

Howell, N. 2010. Life histories of the Dobe!Kung: food, fatness, and well-being over the life span. Berkeley: University of California Press. [MK]

Hrdy, S. B. 1999. Mother Nature: a history of mothers, infants, and natural selection. New York: Pantheon.

- 2009. Mothers and others: the evolutionary origins of mutual understanding. Cambridge, MA: Harvard University Press. [MK]

Ivey, Paula K. 2000. Cooperative reproduction in Ituri Forest hunter-gatherers: who cares for Efé infants? Current Anthropology 41:856-866.

Ivey Henry, Paula, Gilda A. Morelli, and Edward Z. Tronick. 2005. Child caretakers among the Efé foragers of the Ituri Forest. In Hunter-gatherer childhoods: evolutionary, developmental, and cultural perspectives. Michael E. Lamb and Barry S. Hewlett, eds. Pp. 191-123. New York: Aldine de Gruyter. [PIH/GAM/ET]

Jasienska, Grazyna. 2013. The fragile wisdom: an evolutionary view on women's biology and health. Cambridge, MA: Harvard University Press. [PIH]

Kessler, S. E., and L. T. Nash. 2010. Grandmothering in Galago senegalensis braccatus (Senegal Galago). African Primates 7:42-49.

Khatib-Chahidi, J. 1992. Milk kinship in Shi'ite Islamic Iran. In The anthropology of breast-feeding: natural law or social construct. V. Maher, ed. Pp. 109-132. Providence, RI: Berg.

Konner, M. 2005. Hunter-gatherer infancy and childhood: the !Kung and others. In Hunter-gatherer childhoods: evolutionary, developmmental and cultural perspectives. B. S. Hewlett and M. E. Lamb, eds. Pp. 19-64. New Brunswick, NJ: Aldine Transaction. [MK]

- 2010. The evolution of childhood: relationships, emotion, mind. Cambridge, MA: Harvard University Press. [MK]

Konner, M. J., and C. Worthman. 1980. Nursing frequency, gonadal function, and birth spacing among !Kung hunter-gatherers. Science 207:788-791. [AT]

Kramer, K. 2005. Maya children: helpers at the farm. Cambridge, MA: Harvard University Press.

Kramer, K. L., and P. T. Ellison. 2010. Pooled energy budgets: resituating human energy allocation trade-offs. Evolutionary Anthropology 19:136-147.

Kramer, Karen L., and Russell D. Greaves. 2011. Juvenile subsistence effort, activity levels, and growth patterns: middle childhood among Pumé foragers. Human Nature 22(3):303-326. doi:10.1007/s12110-011-9122-8. [PIH/GAM/ET]

Kruger, A. C., and M. Konner. 2010. Who responds to crying? Maternal and allocare among the !Kung. Human Nature-an Interdisciplinary Biosocial Perspective 21(3):309-329. [MK]

Kulski J. K., P. E. Hartmann, W. J. Saint, P. F. Giles, and D. H. Gutteridge. 1981. Changes in the milk composition of non-puerperal women. American Journal of Obstetrics and Gynecology 139:597.

Lachance, Joseph, Benjamin Vernot, Clara C. Elbers, Bart Ferwerda, Alain Froment, Jean-Marie Bodo, Godfrey Lema, Wenqing Fu, Thomas B. Nyambo, Timothy R. Rebbeck, Kun Zhang, Joshua M. Akey, and Sarah A. Tishkoff. 2012. Evolutionary history and adaptation from high-coverage whole-genome sequences of diverse African hunter-gatherers. Cell 150(3): 457-469. doi:10.1016/j.cell.2012.07.009. [PIH/GAM/ET]

Lancaster, J. 1971. Play-mothering: the relations between juvenile females and young infants among free-ranging vervet monkeys (Cercopithecus aethiops). Folia Primatologica 15:161-182.

Lancaster, Jane B., and Hillard S. Kaplan. 1992. Human mating and family formation strategies: the effects of variability among males in quality and the allocation of mating effort and parental investment. In Topics in primatology: human origins. Toshisada Nishida, William C. McGrew, Peter Marler, Martin Pickford, and Frans B. M. de Waal, eds. Pp. 1-14. Tokyo: University of Tokyo Press. [PIH/GAM/ET]

Lanigan, Julie, and Atul Singhal. 2009. Early nutrition and long-term health: a practical approach. Proceedings of the Nutrition Society 68(4):422-429. doi: 10.1017/S002966510999019X. [PIH/GAM/ET]

Lawrence, R. A., and R. M. Lawrence. 2011. Breastfeeding: a guide for the medical profession. Maryland Heights, MO: Elsevier.

Lee, P. C. 1987. Allomothering among African elephants. Animal Behaviour 35:278-291.

Lester, Barry M., Edward Tronick, Eric Nestler, Ted Abel, Barry Kosofsky, Christopher W. Kuzawa, Carmen J. Marsit, et al. 2011. Behavioral epigenetics. Annals of the New York Academy of Sciences 1226(1):14-33. doi: 10.1111/j.1749-6632.2011.06037.x. [PIH/GAM/ET]

Lozoff, B. 1983. Birth and "bonding" in non-industrial societies. Developmental Medicine and Child Neurology 25:595-600.

Macadam, Patricia Stuart, and Katherine A. Dettwyler, eds. 1995. Breastfeeding: biocultural perspectives. Edison, NJ: Transaction. [KH]

Maher, Vanessa, ed. 1992. Anthropology of breast-feeding: natural law or social construct, vol. 3. Oxford: Berg. [KH]

Mann, J., and B. B. Smuts. 1998. Natal attraction: allomaternal care and mother-infant separations in wild bottlenose dolphins. Animal Behaviour 55:1097-1113.

Marlowe, F. 2010. The Hadza: hunter-gatherers of Tanzania. Berkeley: University of California Press. [MK]

Marlowe, F. W. 2003. A critical period for provisioning by Hadza men: implications for pair bonding. Evolution and Human Behavior 24:217-229. [MK]

Martin, Melanie A., and David A. Sela. 2013. Infant gut microbiota: developmental influences and health outcomes. In Building babies. Kathryn B. H. Clancy, Katie Hinde, and Julienne N. Rutherford, eds. Pp. 233-256. New York: Springer. [KH]

Martin, R., M. Olivares, M. Marin, and L. Fernandez. 2005. Probiotic potential of 3 lactobacilli strains isolated from breast milk. Journal of Human Lactation 21:8-17.

McCormick, Marie C., Jeanne Brooks-Gunn, Stephen L. Buka, Julie Goldman, Jennifer Yu, Mikhail Salganik, David T. Scott, et al. 2006. Early intervention in low birth weight premature infants: results at 18 years of age for the infant health and development program. Pediatrics 117(3):771-780. doi: 10.1542/peds.2005-1316. [PIH/GAM/ET]

McDade, Thomas W. 2012. Early environments and the ecology of inflammation. Proceedings of the National Academy of Sciences 109(suppl.):S1728117288. doi:10.1073/pnas.1202244109. [PIH]

McDermott, J., R. Steketee, and J. Wirima. 1996. Perinatal mortality in rural Malawi. Bulletin of the World Health Organization 74(2):165-171. [PIH/ GAM/ET]

McKenna, J. J., S. Mosko, C. Richard, S. Drummond, L. Hunt, M. Cetal, and J. Arpaia. 1994. Mutual behavioral and physiological influences among solitary and co-sleeping mother-infant pairs: implications for SIDS. Early Human Development 38:182-201.

Mead, M. 1930. Growing up in New Guinea. New York: W. Morrow.

Meaney, Michael J., and Moshe Szyf. 2005. Environmental programming of stress responses through DNA methylation: life at the interface between a dynamic environment and a fixed genome. Dialogues in Clinical Neuroscience 7(2):103-123. [PIH/GAM/ET]

Meehan, Courtney L., Robert Quinlan, and Courtney D. Malcom. 2012. Cooperative breeding and maternal energy expenditure among Aka foragers. American Journal of Human Biology 25(1):42-57. doi:10.1002/ajhb.22336. [PIH/GAM/ET]

Mendizabal, Isabel, Urko M. Marigorta, Oscar Lao, and David Comas. 2012. Adaptive evolution of loci covarying with the human African Pygmy phenotype. Human Genetics 131(8):1305-1317. doi:10.1007/s00439-012-11573. [PIH/GAM/ET]

Miller, Elizabeth M., Marco O. Aiello, Masako Fujita, Katie Hinde, Lauren 
Milligan, and E. A. Quinn. 2013. Field and laboratory methods in human milk research. American Journal of Human Biology 25(1):1-11.

Minami, J. 1995. Wet nursing and cross nursing. Leaven 31:53-55.

Morelli, Gilda A., Paula K. Ivey Henry, and Steffen Foerster. Forthcoming. Relationships in a world of uncertainty: dependency and cooperation in the lives of Efé hunter-gatherer infants and toddlers. In Ancestral landscapes in human evolution: culture, childrearing and social wellbeing. Darcia Narvaez, Agustin Fuentes, Kristin Valentino, and Peter B. Gray, eds. Oxford: Oxford University Press. [PIH/GAM/ET]

Morelli, Gilda A., and S. Winn. 1987. Perinatal practices: a biosocial perspective. Advances in Psychology 46:13-22.

Morse, J. M., C. Jehle, and D. Gamble. 1990. Initiating breastfeeding: a world survey of the timing of postpartum breastfeeding. International Journal of Nursing Studies 27:303-313. [ANC]

Neubauer, Simon, and Jean-Jacques Hublin. 2012. The evolution of human brain development. Evolutionary Biology 39(4):568-586. doi:10.1007/ s11692-011-9156-1. [PIH/GAM/ET]

O’Brien, T. G., and J. G. Robinson. 1991. Allomaternal care by female wedgecapped capuchin monkeys: effects of age, rank and relatedness. Behaviour 119:30-50.

Ogunlesi, T. A., F. A. Adekanmbi, B. M. Fetuga, and M. M. Ogundeyi. 2008. Non-puerperal induced lactation: an infant feeding option in paediatric HIV/AIDS in tropical Africa. Journal of Child Health Care 12(3):241-248. [MK]

Packer, C., S. Lewis, and A. Pusey. 1992. A comparative analysis of nonoffspring nursing. Animal Behaviour 43:265-281.

Parkes, P. 2004. Milk kinship in Southeast Europe: alternative social structures and foster relations in the Caucasus and the Balkans. Social Anthropology 12:341-358.

Piperata, Barbara A. 2008. Forty days and forty nights: a biocultural perspective on postpartum practices in the Amazon. Social Science and Medicine 67(7):1094-1103. doi:10.1016/j.socscimed.2008.05.031. [PIH/GAM/ET]

Pusey, A. E., and C. Packer. 1994. Non-offspring nursing in social carnivores: minimizing the costs. Behavioral Ecology 5:362-374.

Ramakrishnan, Usha, Frederick Kobina Grant, Tamar Goldenberg, Vinh Bui, Aamer Imdad, and Zulfiqar Ahmed Bhutta. 2012. Effect of multiple micronutrient supplementation on pregnancy and infant outcomes: a systematic review. Paediatric and Perinatal Epidemiology 26(28):153-167. doi: 10.1111/j.1365-3016.2012.01276.x. [PIH/GAM/ET]

Reiches, Meredith W., Peter T. Ellison, Susan F. Lipson, Katherine C. Sharrock, Eliza Gardiner, and Laura G. Duncan. 2009. Pooled energy budget and human life history. American Journal of Human Biology 21(4):421-429. doi: 10.1002/ajhb.20906. [PIH/GAM/ET]

Richards, A. 1939. Land, labour and diet in Northern Rhodesia: an economic study of the Bemba tribe. London: Oxford University Press.

Richerson, P. J., and R. Boyd. 2004. Not by genes alone. Chicago: University of Chicago Press.

Roulin, A. 2002. Why do lactating females nurse alien offspring? A review of hypotheses and empirical evidence. Animal Behavior 63:201-208.

. 2003. The neuroendocrine function of allosucking. Ethology 109:185195.

Roulin, A., and P. Heeb. 1999. The immunological function of allosucking. Ecology Letters 2:319-324.

Sattenspiel, L. 2000. Tropical environments, human activities, and the transmission of infectious diseases. American Journal of Physical Anthropology 113: 3-31. [ANC]

Scanlon, K. S., M. P. Alexander, M. K. Serdula, M. K. Davis, and B. A. Bowman. 2002. Assessment of infant feeding: the validity of measuring milk intake. Nutrition Reviews 60:235-251. [ANC]

Schuh-Huerta, Sonya M., and Renee A. Reijo Pera. 2011. Reproductive biology: bone returns the favour. Nature 472(7341):46-47. doi:10.1038/ 472046a. [PIH/GAM/ET]

Sear, Rebecca, and Mhairi Gibson. 2009. Introduction to special issue on "Trade-offs in female life histories: integrating evolutionary frameworks." American Journal of Human Biology 21(4):417-420. doi:10.1002/ajhb.20952. [PIH/GAM/ET]

Sear, Rebecca, and Ruth Mace. 2008. Who keeps children alive? A review of the effects of kin on child survival. Evolution and Human Behavior 29(1): 1-18. doi:10.1016/j.evolhumbehav.2007.10.001. [PIH/GAM/ET]

Sellen, Daniel W. 2007. Evolution of infant and young feeding: implications for contemporary public health. Annual Review of Nutrition 27:123-148.
Sirén, Anders, and José Machoa. 2008. Fish, wildlife, and human nutrition in tropical forests: a fat gap? Interciencia 33:186-193. [PIH/GAM/ET]

Slome, C. 1956. Non-puerperal lactation in grandmothers. Journal of Pediatrics 9:550-552.

Super, Charles M., and Sara Harnkness. 2008. Globalization and its discontents: challenges to development theory and practice in Africa. International Journal of Psychology 43(2):107-113. doi:10.1080/00207590701859333. [PIH]

Takada, Akira. 2005. Mother-infant interactions among the !Xun: analysis of gymnastic and breastfeeding behaviors. In Hunter-gatherer childhoods: evolutionary, developmental, and cultural perspectives. B. S. Hewlett and M. E. Lamb, eds. Pp. 289-308. New Brunswick, NJ: Transaction. [AT]

2011. Pre-verbal infant-caregiver interaction. In Handbook of language socialization. A. Duranti, E. Ochs, and B. B. Schieffelin, eds. Pp. 56-80. Oxford: Blackwell. [AT]

Tinbergen, Nicholas. 1963. On aims and methods of ethology, vol. 20. Zeitschrift für Tierpsychologie 20:410-433. [PIH/GAM/ET]

Tronick, Edward Z. 1989. Emotions and emotional communication in infants. American Psychologist 44(2):112-119. doi:10.1037/0003-066X.44.2.112. [PIH/GAM/ET]

Tronick, Edward Z., Gilda A. Morelli, and Paula K. Ivey. 1992. The Efé forager infant and toddler's pattern of social relationships: multipe and simultaneous. Developmental Psychology 28(4):568-577. doi:10.1037/0012-1649 .28.4.568. [PIH]

Tronick, Edward Z., Gilda A. Morelli, and Steve A. Winn. 1987. Multiple caretaking of Efé (Pygmy) infants. American Anthropologist 87:96-106. doi: 10.1525/aa.1987.89.1.02a00050.

_ 1989. The caretaker-child strategic model: Efé and Aka child rearing as exemplars of the multiple factors affecting child rearing-a reply to Hewlett. American Anthropologist 91:192-194. doi:10.2307/679754. [PIH]

Tronick, Edward Z., and Steve A. Winn. 1992. The neruobehavioral organization of Efé (Pygmy) infants. Journal of Developmental and Behavioral Pediatrics 13(6):421-424.

Turke, P. W. 1988. Helpers at the nest: childcare networks on Ifaluk. In Human reproductive behavior: a Darwinian perspective. L. Betzig, M. Borgerhoff Mulder, and P. Turke, eds. Pp. 173-188. Cambridge: Cambridge University Press.

Valeggia, Claudia, and Peter T. Ellison. 2009. Interactions between metabolic and reproductive functions in the resumption of postpartum fecundity. American Journal of Human Biology 21(4):559-566. [KH]

Van Esterik, Penny. 2002. Contemporary trends in infant feeding research. Annual Review of Anthropology 31:257-278. [KH]

Verdu, Paul P., and Giovanni G. Destro-Bisol. 2012. African Pygmies, what's behind a name? Human Biology 84(1):1-10. doi:10.3378/027.084.0105. [PIH/GAM/ET]

Wells, J. 2006. The role of cultural factors in human breastfeeding. Special issue, Journal of Human Ecology 14:39-47.

Wells, Jonathan C. K. 2007. The programming effects of early growth. Early Human Development 83(12):743-748. [KH]

. 2011. The thrifty phenotype: an adaptation in growth or metabolism? American Journal of Human Biology 23(1):65-75. [KH]

Winn, Steve A., Gilda A. Morelli, and Edward Z. Tronick. 1987. The infant and the group: a look at Efé caretaking practices. In The cultural context of infancy. J. K. Nugent, B. M. Lester, and T. E. Brazelton, eds. Pp. 87-110. Norwood, NJ: Ablex.

Work, T. H. A., A. Ifekwunigwe, D. B. Jeliffe, P. Jeliffe, and C. G. Neumann. 1973. Tropical problems in nutrition. Annals of Internal Medicine 79:701711. [ANC]

World Health Organization. 1998. Relactation: a review of experience and recommendations for practice. Geneva: World Health Organization

Zerfu, Taddese Alemu, and Henok Taddese Ayele. 2013. Micronutrients and pregnancy effect of supplementation on pregnancy and pregnancy outcomes: a systematic review. Nutrition Journal 12(1):20-25. doi:10.1186/ 1475-2891-12-20. [PIH/GAM/ET]

Zimmermann, Michael B., and Maria Andersson. 2012. Assessment of iodine nutrition in populations: past, present, and future. Nutrition Reviews 70(10): 553-570. doi:10.1111/j.1753-4887.2012.00528.x. [PIH/GAM/ET]

Zureick-Brown, Sarah, Holly Newby, Doris Chou, Nobuko Mizoguchi, Lale Say, Emi Suzuki, and John Wilmoth. 2013. Understanding global trends in maternal mortality. International Perspectives on Sexual and Reproductive Health 39(1):32-41. [PIH/GAM/ET] 